Supporting information for

\title{
Synthesis of Novel polycyclic Indolyldiamines
}

Sabrina Cutri, $\S$ Anna Diez, ${ }^{*}{ }^{*}$ Martine Bonin,*\$ Laurent Micouin ${ }^{\S}$ and Henri-Philippe Husson $^{\S}$

Laboratoire de Chimie Thérapeutique, UMR 8638 associée au CNRS et à l'Université René Descartes, Faculté des Sciences Pharmaceutiques et Biologiques, 4 av de l'Observatoire, 75270 Paris cedex 06 (France) and Parc Científic de Barcelona c/Josep Samitier, 1-5 08028-Barcelona (Spain).

martine.bonin@univ-paris5.fr

General methods

Silica gel (230-400 mesh) was used for product purification by flash column chromatography. Solvents were purified and dried by standard techniques, and distilled prior to use. All organic extracts were dried over $\mathrm{MgSO}_{4}$ or $\mathrm{Na}_{2} \mathrm{SO}_{4}$. IR spectra were recorded on a Perkin-Elmer FTIR 1600 spectrometer as thin film. ${ }^{1} \mathrm{H}$ and ${ }^{13} \mathrm{C}$ NMR spectra were recorded on a Bruker AC-300 or AC-400 spectrometer with TMS as internal standard and $\mathrm{CDCl}_{3}$ as solvent. HRMS were determined on a Kratos MS 80 RF or Micromass Q Tof 1 spectrometer.

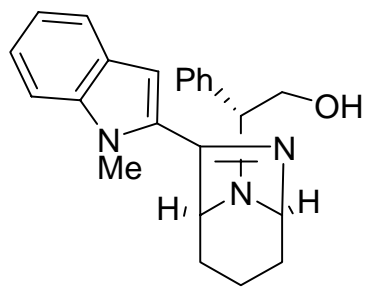

2-[7-(1-Methyl-1H-indol-2-yl)-6,8-diazabicyclo[3.2.1] oct-6-en-8-yl]-2-phenylethanol 6

To a stirred solution of TMEDA ( 2 eq., $44 \mathrm{mmol}, 6.62 \mathrm{~mL}$ ) was added $n$-BuLi ( 2 eq., $44 \mathrm{mmol}, 1.6 \mathrm{M}, 27.5 \mathrm{~mL}$ ) under argon at $-20{ }^{\circ} \mathrm{C}$. After $5 \mathrm{~min}$. stirring, N-methylindole (3 eq., $66 \mathrm{mmol}, 8.42 \mathrm{~mL}$ ) was dropwise added. Stirring was maintained for $1 \mathrm{~h}$ while proceeding to two ultrasonic irradiations (2 x 1 min.). A solution of (-)-2-cyano-6phenyloxazolopiperidine 1 ( $22 \mathrm{mmol}, 5 \mathrm{~g})$ in anhydrous ether $(200 \mathrm{~mL})$ was then added under argon. Stirring was maintained for $5 \mathrm{~h}$ until the temperature of the reaction mixture was warmed gradually to room temperature. The reaction mixture was cautiously diluted with a saturated, aqueous solution of $\mathrm{NH}_{4} \mathrm{Cl}$, extracted with $\mathrm{CH}_{2} \mathrm{Cl}_{2}(2 \times 150 \mathrm{~mL})$, and the combined organic layers were dried $\left(\mathrm{MgSO}_{4}\right)$. The solvents were evaporated to give an oily residue which was purified by chromatography $\left(\mathrm{CH}_{2} \mathrm{Cl}_{2} / \mathrm{MeOH} 98 / 2\right.$ then $\left.95 / 5\right)$ to provide imine 6 (yellow oil, $7.27 \mathrm{~g}, 92 \%$ ). [ $\alpha]_{\mathrm{D}}{ }^{20}-197$ (c 1.0, MeOH); IR (KBr) $v_{\max } 3350,3027,2990,2855$, 1648, 1610, 1468, 1087, $1059 \mathrm{~cm}^{-1} ;{ }^{1} \mathrm{H}$ NMR $\left(\mathrm{CDCl}_{3}, 300 \mathrm{MHz}\right): \delta 7.56(\mathrm{~d}, J=8.0 \mathrm{~Hz}, \mathrm{CH})$, 7.20-7.40 (7 H, m, 7CH), $7.10\left(1 \mathrm{H}, \mathrm{td}, J_{1}=7.8 \mathrm{~Hz}, J_{2}=1.2 \mathrm{~Hz}, \mathrm{CH}\right), 6.56(1 \mathrm{H}, \mathrm{s}, \mathrm{CH}), 5.54$ $(1 \mathrm{H}, \mathrm{t}, J=2.4 \mathrm{~Hz}, \mathrm{CH}), 4.09\left(3 \mathrm{H}, \mathrm{s}, \mathrm{CH}_{3}\right), 3.98(1 \mathrm{H}, \mathrm{t}, J=3.0 \mathrm{~Hz}, \mathrm{CH}), 3.94\left(1 \mathrm{H}, \mathrm{dd}, J_{l}=\right.$ $\left.11.1 \mathrm{~Hz}, J_{2}=5.4 \mathrm{~Hz}, \mathrm{CH}_{2}\right), 3.81\left(1 \mathrm{H}, \mathrm{dd}, J_{1}=11.1 \mathrm{~Hz}, J_{2}=5.4 \mathrm{~Hz}, \mathrm{CH}_{2}\right), 3.50(1 \mathrm{H}, \mathrm{t}, J=$ $5.4 \mathrm{~Hz}, \mathrm{CH}), 1.20-1.90\left(7 \mathrm{H}, \mathrm{m}, 3 \mathrm{CH}_{2}, \mathrm{OH}\right) ;{ }^{13} \mathrm{C} \mathrm{NMR}\left(\mathrm{CDCl}_{3}, 75 \mathrm{MHz}\right): \delta 165.3,140.2$, 131.0, 128.7, 128.5, 127.9, 126.8, 121.7, 124.4, 120.2, 110.0, 108.8, 87.0, 66.1, 67.6, 64.3, 32.3, 16.9, 25.5, 26.1; MS (CI) m/z $360\left(\mathrm{MH}^{+}\right)$; HRMS (CI) calcd for $(\mathrm{MH})^{+}, 360.2076$, found for $(\mathrm{MH})^{+}: 360.2074$. 


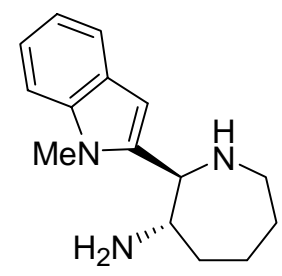

2-(1-Methyl-1H-indol-2-yl)-azepan-3ylamine 7

$\mathrm{LiAlH}_{4}$ (8 eq., $71.4 \mathrm{mmol}, 2.72 \mathrm{~g}$ ) was carefully added at $0{ }^{\circ} \mathrm{C}$ to a solution of imine $6(8.92 \mathrm{mmol}, 3.20 \mathrm{~g})$ in dry $i \mathrm{Pr}_{2} \mathrm{O}(160 \mathrm{~mL})$ under argon. The reaction mixture was stirred for $24 \mathrm{~h}$ at reflux, allowed to cool and treated at $0{ }^{\circ} \mathrm{C}$ successively with aqueous $\mathrm{NaOH}$ $(1 \mathrm{M}, 5.4 \mathrm{~mL})$ and $\mathrm{H}_{2} \mathrm{O}(8.2 \mathrm{~mL})$. After filtration through celite, the residue was washed several times with $\mathrm{Et}_{2} \mathrm{O}$ and the organic layer was concentrated in vacuo. The crude $(3.24 \mathrm{~g})$ was then dissolved in methanol $(120 \mathrm{~mL})$ and stirred under hydrogen in presence of aqueous $\mathrm{HCl}(12 \mathrm{~N}, \mathrm{pH} 2)$ and $10 \% \mathrm{Pd} / \mathrm{C}(647 \mathrm{mg})$ for $48 \mathrm{~h}$ at room temperature. After filtration through celite and concentration in vacuo, the residue was dissolved in ether and extracted three times with an aqueous $2 \mathrm{~N} \mathrm{HCl}$ solution. Aqueous layers were made basic $(\mathrm{NaOH} 6 \mathrm{~N})$ and then extracted with AcOEt. The organic layers were dried $\left(\mathrm{Na}_{2} \mathrm{SO}_{4}\right)$ and concentrated to provide an oily residue. The residue was purified by chromatography (AcOEt/MeOH/NH$/ \mathrm{OH}$ $175 / 20 / 5)$ to provide azepane 7 (yellow oil, $1.76 \mathrm{~g}, 81 \%)$. [ $\alpha]_{\mathrm{D}}{ }^{20}-3\left(\mathrm{c} 0.7, \mathrm{CHCl}_{3}\right)$; IR $(\mathrm{KBr})$ $v_{\max } 3360,2928,1468,1315,1135 \mathrm{~cm}^{-1} ;{ }^{1} \mathrm{H} \mathrm{NMR}\left(\mathrm{CDCl}_{3}, 300 \mathrm{MHz}\right): \delta 7.56(1 \mathrm{H}, \mathrm{d}, J=7.3$ $\mathrm{Hz}, \mathrm{CH}), 7.30(1 \mathrm{H}, \mathrm{d}, J=8.2 \mathrm{~Hz}, \mathrm{CH}), 7.20\left(1 \mathrm{H}, \mathrm{td}, J_{1}=7.7 \mathrm{~Hz}, J_{2}=0.7 \mathrm{~Hz}, \mathrm{CH}\right), 7.08(1$ $\left.\mathrm{H}, \mathrm{td}, J_{1}=7.2 \mathrm{~Hz}, J_{2}=0.6 \mathrm{~Hz}, \mathrm{CH}\right), 6.44(1 \mathrm{H}, \mathrm{s}, \mathrm{CH}), 3.80\left(3 \mathrm{H}, \mathrm{s}, \mathrm{CH}_{3}\right), 3.60(1 \mathrm{H}, \mathrm{d}, J=$ $9.6 \mathrm{~Hz}, \mathrm{CH}), 3.39(1 \mathrm{H}, \mathrm{m}, \mathrm{CH}), 3.15\left(1 \mathrm{H}, \mathrm{dt}, J_{l}=13.7 \mathrm{~Hz}, J_{2}=4.9 \mathrm{~Hz}, \mathrm{CH}_{2}\right), 2.84(1 \mathrm{H}, \mathrm{m}$, $\left.\mathrm{CH}_{2}\right), 1.60-2.10\left(9 \mathrm{H}, \mathrm{m}, 3 \mathrm{CH}_{2}, \mathrm{NH}, \mathrm{NH}_{2}\right) ;{ }^{13} \mathrm{C} \mathrm{NMR}\left(\mathrm{CDCl}_{3}, 75 \mathrm{MHz}\right): \delta 142.3,137.8$, 127.6, 121.5, 120.5, 119.6, 109.2, 98.9, 65.0, 56.8, 48.6, 35.5, 30.7, 30.2, 22.4; MS (CI) m/z $244\left(\mathrm{MH}^{+}\right)$; HRMS (CI) calcd for $(\mathrm{MH})^{+}, 244.1814$, found for $(\mathrm{MH})^{+}: 244.1816$.

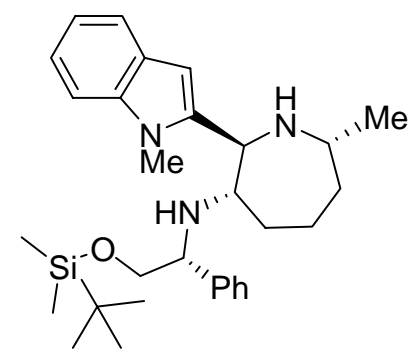

[2-(tert-Butyl-dimethyl-silanyloxy)-1phenyl-ethyl]-[7-methyl-2-(1-methyl-1Hindol-2-yl)-azepan-3-yl]-amine 9

$\mathrm{NaH}(60 \%$ suspension in mineral oil, 2 eq., $2.03 \mathrm{mmol}, 81 \mathrm{mg})$ was added at $0{ }^{\circ} \mathrm{C}$ under argon to a solution of imine $6(1.02 \mathrm{mmol}, 365 \mathrm{mg})$ in THF $(15 \mathrm{~mL})$. After $30 \mathrm{~min}$. at room temperature, tert-butyldimethylsilyl chloride (2 eq., $2.03 \mathrm{mmol}, 307 \mathrm{mg}$ ) was added. The resulting mixture was stirred for an additional $16 \mathrm{~h}$ at $20{ }^{\circ} \mathrm{C}$. The reaction mixture was cautiously diluted with a saturated, aqueous $\mathrm{NH}_{4} \mathrm{Cl}$ solution and extracted with $\mathrm{CH}_{2} \mathrm{Cl}_{2}(2 \mathrm{x})$. The combined organic layers were dried $\left(\mathrm{MgSO}_{4}\right)$ and concentrated in vacuo. The oily residue $(600 \mathrm{mg})$ was then dissolved in $\mathrm{Et}_{2} \mathrm{O}(20 \mathrm{~mL}) . \mathrm{LiAlH}_{4}(5 \mathrm{eq} ., 5.10 \mathrm{mmol}, 194 \mathrm{mg})$ was carefully added at $0{ }^{\circ} \mathrm{C}$ under argon. The reaction mixture was stirred for $1 \mathrm{~h} 30$ at $0{ }^{\circ} \mathrm{C}$, and treated successively with aqueous $\mathrm{NaOH}(1 \mathrm{M}, 389 \mu \mathrm{L})$ and $\mathrm{H}_{2} \mathrm{O}(583 \mu \mathrm{L})$. After filtration through celite the residue was washed several times with $\mathrm{Et}_{2} \mathrm{O}$ and the organic layer was concentrated in vacuo providing the aminal 8 as colourless oil $(550 \mathrm{mg})$. This aminal was dissolved in $\mathrm{Et}_{2} \mathrm{O}(24 \mathrm{~mL})$ under argon. An etheral solution of methylmagnesium bromide (3 
$\mathrm{M} / \mathrm{Et}_{2} \mathrm{O}, 3.5$ eq., $3.57 \mathrm{mmol}, 1.19 \mathrm{~mL}$ ) was carefully added at room temperature. The reaction mixture was stirred for $3 \mathrm{~h}$ at reflux. The reaction mixture was cautiously treated with a saturated, aqueous solution of $\mathrm{NH}_{4} \mathrm{Cl}$, extracted with $\mathrm{CH}_{2} \mathrm{Cl}_{2}(2 \times 40 \mathrm{~mL})$. The combined organic layers were dried $\left(\mathrm{MgSO}_{4}\right)$. The solvents were evaporated to give an oily residue. The residue was purified by chromatography $\left(\mathrm{AcOEt} / \mathrm{CH}_{3} \mathrm{CN} / \mathrm{NH}_{4} \mathrm{OH} 195 / 4 / 1\right)$ to provide azepane 9 (yellow oil, $176 \mathrm{mg}, 35 \%$ ). [ $\alpha]_{\mathrm{D}}{ }^{20}-26\left(\mathrm{c} 1.4, \mathrm{CH}_{2} \mathrm{Cl}_{2}\right)$; IR $(\mathrm{KBr}) v_{\max } 2952,2926$, 2855, 1470, 1314, 1255, $1091 \mathrm{~cm}^{-1} ;{ }^{1} \mathrm{H} \mathrm{NMR}\left(\mathrm{CDCl}_{3}, 400 \mathrm{MHz}\right): \delta 7.64(1 \mathrm{H}, \mathrm{d}, J=7.7 \mathrm{~Hz}$, $\mathrm{CH}), 7.22-7.44(7 \mathrm{H}, \mathrm{m}, 7 \mathrm{CH}), 7.15(1 \mathrm{H}, \mathrm{t}, J=7.6 \mathrm{~Hz}, \mathrm{CH}), 6.56(1 \mathrm{H}, \mathrm{s}, \mathrm{CH}), 4.18(1 \mathrm{H}, \mathrm{d}, J$ $=7.6 \mathrm{~Hz}, \mathrm{CH}), 3.86\left(3 \mathrm{H}, \mathrm{s}, \mathrm{CH}_{3}\right), 3.70\left(1 \mathrm{H}, \mathrm{dd}, J_{l}=8.0 \mathrm{~Hz}, J_{2}=4.2 \mathrm{~Hz}, \mathrm{CH}\right), 3.58(1 \mathrm{H}, \mathrm{dd}$, $\left.J_{1}=10.0 \mathrm{~Hz}, J_{2}=4.2 \mathrm{~Hz}, \mathrm{CH}_{2}\right), 3.52\left(1 \mathrm{H}, \mathrm{t}, J=9.0 \mathrm{~Hz}, \mathrm{CH}_{2}\right), 3.26\left(1 \mathrm{H}, \mathrm{td}, J_{1}=7.6 \mathrm{~Hz}, J_{2}=\right.$ $2.1 \mathrm{~Hz}, \mathrm{CH}), 2.90\left(1 \mathrm{H}, \mathrm{m}, \mathrm{CH}_{2}\right), 2.48(2 \mathrm{H}, \mathrm{bs}, 2 \mathrm{NH}), 1.18-1.94\left(6 \mathrm{H}, \mathrm{m}, 3 \mathrm{CH}_{2}\right), 0.95(3 \mathrm{H}, \mathrm{d}$, $\left.J=6.4 \mathrm{~Hz}, \mathrm{CH}_{3}\right), 0.74\left(9 \mathrm{H}, \mathrm{s}, 3 \mathrm{CH}_{3}\right),-0.10\left(3 \mathrm{H}, \mathrm{s}, \mathrm{CH}_{3}\right),-0.13\left(3 \mathrm{H}, \mathrm{s}, \mathrm{CH}_{3}\right) ;{ }^{13} \mathrm{C} \mathrm{NMR}$ $\left(\mathrm{CDCl}_{3}, 75 \mathrm{MHz}\right): \delta 142.1,141.5,137.9,128.3,127.9,127.6,127.4,121.2,120.6,119.3$, 109.1, 99.9, 68.0, 64.0, 62.2, 56.8, 48.3, 38.5, 33.9, 30.1, 25.8, 24.7, 24.5, 18.2, 1.9, 1.6; MS (CI) $\mathrm{m} / \mathrm{z} 492.3\left(\mathrm{MH}^{+}\right)$; HRMS (CI) calcd for $(\mathrm{MH})^{+}, 492.3410$, found for $(\mathrm{MH})^{+}: 492.3411$.

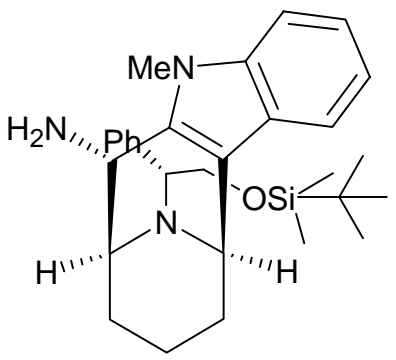

Tetracycle $\mathbf{1 0}$

Aminal $8(550 \mathrm{mg})$ was dissolved in ethyl acetate $(19.6 \mathrm{~mL})$ at room temperature. $\mathrm{CH}_{3} \mathrm{CN}(0.4 \mathrm{~mL})$ and silica $(3 \mathrm{~g})$ were added. The reaction mixture was stirred for $4 \mathrm{~h}$ at room temperature and filtered. The organic layer was concentrated in vacuo give an oily residue. The residue was purified by chromatography $\left(\mathrm{AcOEt} / \mathrm{CH}_{3} \mathrm{CN} 95 / 5\right)$ to provide tetracycle 10 (yellow oil, $102 \mathrm{mg}, 21 \%$ from 6). $[\alpha]_{\mathrm{D}}{ }^{20}+61\left(\mathrm{c} 0.7, \mathrm{CH}_{2} \mathrm{Cl}_{2}\right)$; IR $(\mathrm{KBr}) v_{\max } 2928,2855$, 1470, 1372, 1254, 1190, 1096, $1007 \mathrm{~cm}^{-1} ;{ }^{1} \mathrm{H} \mathrm{NMR}\left(\mathrm{CDCl}_{3}, 300 \mathrm{MHz}\right): \delta 7.40\left(1 \mathrm{H}, \mathrm{dd}, J_{l}=\right.$ $\left.7.8 \mathrm{~Hz}, J_{2}=1.6 \mathrm{~Hz}, \mathrm{CH}\right), 7.20-7.32(6 \mathrm{H}, \mathrm{m}, 6 \mathrm{CH}), 7.14\left(1 \mathrm{H}, \mathrm{td}, J_{l}=7.5 \mathrm{~Hz}, J_{2}=1.2 \mathrm{~Hz}\right.$, $\mathrm{CH}), 6.98\left(1 \mathrm{H}, \mathrm{td}, J_{1}=7.1 \mathrm{~Hz}, J_{2}=0.9 \mathrm{~Hz}, \mathrm{CH}\right), 4.32(1 \mathrm{H}, \mathrm{t}, J=4.8 \mathrm{~Hz}, \mathrm{CH}), 4.06(1 \mathrm{H}, \mathrm{bs}$, $\mathrm{CH}), 3.83\left(3 \mathrm{H}, \mathrm{s}, \mathrm{CH}_{3}\right), 3.80-3.87\left(3 \mathrm{H}, \mathrm{m}, \mathrm{CH}, \mathrm{CH}_{2}\right), 3.57(1 \mathrm{H}, \mathrm{d}, \mathrm{J}=5.4 \mathrm{~Hz}, \mathrm{CH}), 1.89$ (2 $\left.\mathrm{H}, \mathrm{bs}, \mathrm{NH}_{2}\right), 1.13-2.20\left(6 \mathrm{H}, \mathrm{m}, 3 \mathrm{CH}_{2}\right), 0.82\left(9 \mathrm{H}, \mathrm{s}, 3 \mathrm{CH}_{3}\right),-0.12\left(3 \mathrm{H}, \mathrm{s}, \mathrm{CH}_{3}\right),-0.14(3 \mathrm{H}, \mathrm{s}$, $\left.\mathrm{CH}_{3}\right) ;{ }^{13} \mathrm{C}$ NMR $\left(\mathrm{CDCl}_{3}, 75 \mathrm{MHz}\right): \delta 142.6,138.3,137.4,128.4,127.2,124.4,121.0,118.6$, 118.4, 112.4, 109.0, 67.8, 64.9, 59.3, 50.0, 48.6, 29.7, 26.0, 24.6, 22.2, 18.3, 16.8, 1.5; MS (CI) $\mathrm{m} / \mathrm{z} 476.3\left(\mathrm{MH}^{+}\right)$; HRMS (CI) calcd for $(\mathrm{MH})^{+}, 476.3097$, found for $(\mathrm{MH})^{+}: 476.3098$.

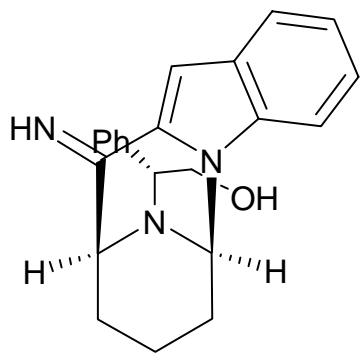

Tetracycle 11 
To a stirred solution of diisopropylamine (2 eq., $1.75 \mathrm{mmol}, 247 \mu \mathrm{L})$ in THF $(1 \mathrm{~mL})$ was added $n$-BuLi (1.1 eq., $0.965 \mathrm{mmol}, 2.5 \mathrm{M}, 386 \mu \mathrm{L}$ ) under argon at $-70{ }^{\circ} \mathrm{C}$. After being stirred for $30 \mathrm{~min}$. at $-70{ }^{\circ} \mathrm{C}$, the reaction mixture was warmed gradually to $0{ }^{\circ} \mathrm{C}$. A solution of N-phenylsulfonylindole (2.5 eq., $2.19 \mathrm{mmol}, 563 \mathrm{mg})$ in THF $(1 \mathrm{~mL})$ was added dropwise. Stirring was maintained for $30 \mathrm{~min}$. before cooling the reaction mixture to $-70{ }^{\circ} \mathrm{C}$. A solution of (-)-2-cyano-6-phenyloxazolopiperidine $1(0.877 \mathrm{mmol}, 200 \mathrm{mg})$ in THF $(1 \mathrm{~mL})$ was then added under argon. Stirring was maintained for $3 \mathrm{~h}$ until the temperature of the reaction mixture was warmed gradually to room temperature. The reaction mixture was cautiously treated with a saturated, aqueous solution of $\mathrm{NH}_{4} \mathrm{Cl}$, extracted with $\mathrm{CH}_{2} \mathrm{Cl}_{2}(2 \times 50 \mathrm{~mL})$, and the combined organic layers were dried $\left(\mathrm{MgSO}_{4}\right)$. The solvents were evaporated to give an oily residue. The residue was purified by chromatography $\left(\mathrm{CH}_{2} \mathrm{Cl}_{2} / \mathrm{MeOH} 98 / 2\right)$ to provide imine 10 (yellow oil, $121 \mathrm{mg}, 40 \%$ ). $[\alpha]_{\mathrm{D}}{ }^{20}-37\left(\mathrm{c} 0.8, \mathrm{CH}_{2} \mathrm{Cl}_{2}\right)$; IR $(\mathrm{KBr}) v_{\max } 3266,3061$, 2948, 2872, 1607, 1454, 1420, 1349, 1333, 1310, 1265, 1082, $1053 \mathrm{~cm}^{-1} ;{ }^{1} \mathrm{H} \mathrm{NMR}\left(\mathrm{CDCl}_{3}\right.$, $200 \mathrm{MHz}): \delta 7.54(1 \mathrm{H}, \mathrm{d}, J=8.1 \mathrm{~Hz}, \mathrm{CH}), 7.15-7.31(7 \mathrm{H}, \mathrm{m}, 7 \mathrm{CH}), 7.09(1 \mathrm{H}, \mathrm{m}, \mathrm{CH}), 6.55$ $(1 \mathrm{H}, \mathrm{s}, \mathrm{CH}), 5.52(1 \mathrm{H}, \mathrm{bs}, \mathrm{CH}), 4.45(1 \mathrm{H}, \mathrm{bs}, \mathrm{NH}), 3.98\left(2 \mathrm{H}, \mathrm{m}, \mathrm{CH}_{2}\right), 3.89(1 \mathrm{H}, \mathrm{bs}, \mathrm{CH})$, $3.43(1 \mathrm{H}, \mathrm{t}, J=5.1 \mathrm{~Hz}, \mathrm{CH}), 1.10-1.94\left(7 \mathrm{H}, \mathrm{m}, 3 \mathrm{CH}_{2}, \mathrm{OH}\right) ;{ }^{13} \mathrm{C} \mathrm{NMR}\left(\mathrm{CDCl}_{3}, 50 \mathrm{MHz}\right)$ : $\delta 165.3,140.3,137.7,129.6,128.6,128.4,127.7,124.8,121.6,120.3,112.0,108.5,85.8$, 66.7, 66.6, 65.4, 25.9, 25.4, 16.7; MS (EI) m/z $345\left(\mathrm{M}^{+}\right)$; HRMS (CI) calcd for $(\mathrm{M})^{+}$, 345.1841, found for $(\mathrm{M})^{+}: 345.1843$.

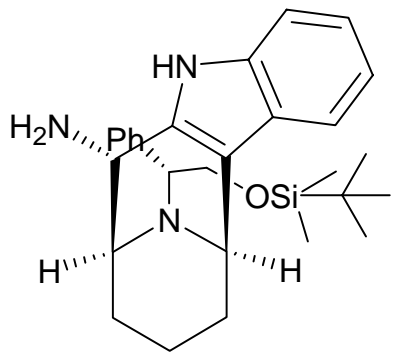

Tetracycle 12

$\mathrm{LiAlH}_{4}$ (4 eq., $1.16 \mathrm{mmol}, 44 \mathrm{mg}$ ) was carefully added at $-20{ }^{\circ} \mathrm{C}$ to a solution of imine $11(0.290 \mathrm{mmol}, 100 \mathrm{mg})$ in anhydrous $\mathrm{Et}_{2} \mathrm{O}(5 \mathrm{~mL})$ under argon. The reaction mixture was stirred for $3 \mathrm{~h}$ and the temperature reaction was gradually warmed to room temperature. The reaction mixture was treated successively with aqueous $\mathrm{NaOH}(1 \mathrm{M}, 88 \mu \mathrm{L})$ and $\mathrm{H}_{2} \mathrm{O}$ $(132 \mu \mathrm{L})$. After filtration through celite the residue was washed several times with $\mathrm{Et}_{2} \mathrm{O}$ and the organic layer was concentrated in vacuo to give an oily residue. The residue was purified by chromatography (AcOEt/MeOH/ $\mathrm{NH}_{4} \mathrm{OH} 179 / 20 / 1$ ) to provide tetracycle 12 (yellow oil, 38 $\mathrm{mg}, 38 \%)$. $[\alpha]_{\mathrm{D}}{ }^{20}-4\left(\mathrm{c} 0.8, \mathrm{CH}_{2} \mathrm{Cl}_{2}\right)$; IR $(\mathrm{KBr}) v_{\max } 3395,2932,1639,1453,1341,1265$, 1237, $1050 \mathrm{~cm}^{-1} ;{ }^{1} \mathrm{H} \mathrm{NMR}\left(\mathrm{CDCl}_{3}, 300 \mathrm{MHz}\right): \delta 9.00(1 \mathrm{H}, \mathrm{bs}, \mathrm{NH}), 7.19-7.42(7 \mathrm{H}, \mathrm{m}$, $7 \mathrm{CH}), 7.13\left(1 \mathrm{H}, \mathrm{td}, J_{l}=7.4 \mathrm{~Hz}, J_{2}=1.1 \mathrm{~Hz}, \mathrm{CH}\right), 7.04\left(1 \mathrm{H}, \mathrm{td}, J_{1}=7.4 \mathrm{~Hz}, J_{2}=1.0 \mathrm{~Hz}\right.$, $\mathrm{CH}), 4.34(1 \mathrm{H}, \mathrm{bs}, \mathrm{CH}), 4.07(1 \mathrm{H}, \mathrm{t}, J=4.9 \mathrm{~Hz}, \mathrm{CH}), 3.92\left(1 \mathrm{H}, \mathrm{dd}, J_{l}=11.1 \mathrm{~Hz}, J_{2}=4.9\right.$ $\left.\mathrm{Hz}, \mathrm{CH}_{2}\right), 3.66\left(2 \mathrm{H}, \mathrm{m}, \mathrm{CH}, \mathrm{CH}_{2}\right), 3.23(1 \mathrm{H}, \mathrm{bd}, J=4.0 \mathrm{~Hz}, \mathrm{CH}), 2.30\left(2 \mathrm{H}, \mathrm{bs}, \mathrm{NH}_{2}\right), 1.12-$ $2.02\left(7 \mathrm{H}, \mathrm{m}, 3 \mathrm{CH}_{2}, \mathrm{OH}\right) \mathrm{ppm} ;{ }^{13} \mathrm{C} \mathrm{NMR}\left(\mathrm{CDCl}_{3}, 75 \mathrm{MHz}\right): \delta 141.5,136.4,136.2,128.8$, 128.5, 127.7, 125.3, 121.7, 119.3, 118.2, 112.2, 111.3, 64.9, 64.7, 59.6, 49.7, 48.4, 28.0, 26.2, $16.6 \mathrm{ppm}, \mathrm{MS}(\mathrm{CI}) \mathrm{m} / \mathrm{z} 348\left(\mathrm{MH}^{+}\right)$; HRMS $(\mathrm{CI})$ calcd for $(\mathrm{MH})^{+}, 348.2076$, found for $(\mathrm{MH})^{+}$: 348.2075 . 


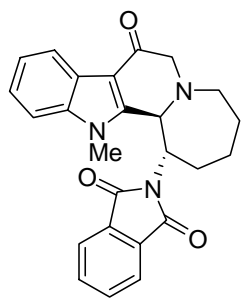

Tetracycle 14

To a solution of azepane $7(2.14 \mathrm{mmol}, 519 \mathrm{mg})$ in THF $(10 \mathrm{~mL})$, distilled $\mathrm{Et}_{3} \mathrm{~N}$ (1.5 eq., $3.21 \mathrm{mmol}, 638 \mu \mathrm{L})$ and $\mathrm{N}$-carbethoxyphtalimide $(1.5$ eq., $3.21 \mathrm{mmol}, 701 \mathrm{mg}$ ) were added at room temperature under nitrogen. The reaction mixture was stirred for $24 \mathrm{~h}$ at reflux, cautiously diluted with $\mathrm{H}_{2} \mathrm{O}$, extracted with $\mathrm{CH}_{2} \mathrm{Cl}_{2}(2 \times 100 \mathrm{~mL})$, and the combined organic layers were dried $\left(\mathrm{MgSO}_{4}\right)$. The solvents were evaporated to give an oily residue. The crude was diluted in $\mathrm{CH}_{2} \mathrm{Cl}_{2}(16 \mathrm{~mL})$. Glyoxal (40\% in $\mathrm{H}_{2} \mathrm{O}, 3$ eq., $\left.6.43 \mathrm{mmol}, 736 \mu \mathrm{L}\right)$ and acetic acid (4 eq., $8.58 \mathrm{mmol}, 488 \mu \mathrm{L}$ ) were added at room temperature. Stirring was maintained for $5 \mathrm{~h}$. The reaction mixture was cautiously diluted with a saturated, aqueous solution of $\mathrm{NaHCO}_{3}$, extracted with $\mathrm{CH}_{2} \mathrm{Cl}_{2}(2 \times 10 \mathrm{~mL})$, and the combined organic layers were dried $\left(\mathrm{MgSO}_{4}\right)$. The solvents were evaporated to give an oily residue. The residue was purified by chromatography $\left(\mathrm{CH}_{2} \mathrm{Cl}_{2}\right.$ then $\mathrm{CH}_{2} \mathrm{Cl}_{2} / \mathrm{MeOH} 99 / 1$ and finally $\left.\mathrm{CH}_{2} \mathrm{Cl}_{2} / \mathrm{MeOH} 96 / 4\right)$ to provide ketone 14 (yellow oil, $574 \mathrm{mg}, 65 \%)$. [ $\alpha]_{\mathrm{D}}{ }^{20}+470\left(\mathrm{c} 0.9, \mathrm{CH}_{2} \mathrm{Cl}_{2}\right)$; IR $(\mathrm{KBr}) v_{\max }$ 2927, 2861, 1771, 1713, 1652, 1614, 1480, 1464, 1422, 1391, 1357, 1329, 1132, 1090, 1063 $\mathrm{cm}^{-1} ;{ }^{1} \mathrm{H} \mathrm{NMR}\left(\mathrm{CDCl}_{3}, 300 \mathrm{MHz}\right): \delta 8.23(1 \mathrm{H}, \mathrm{d}, J=7.5 \mathrm{~Hz}, \mathrm{CH}), 7.90(1 \mathrm{H}, \mathrm{m}, \mathrm{CH}), 7.70$ $(3 \mathrm{H}, \mathrm{m}, 3 \mathrm{CH}), 7.26\left(1 \mathrm{H}, \mathrm{td}, J_{1}=7.4 \mathrm{~Hz}, J_{2}=1.0 \mathrm{~Hz}, \mathrm{CH}\right), 7.18\left(1 \mathrm{H}, \mathrm{td}, J_{l}=7.6 \mathrm{~Hz}, J_{2}=\right.$ $1.2 \mathrm{~Hz}, \mathrm{CH}), 7.03(1 \mathrm{H}, \mathrm{d}, J=7.6 \mathrm{~Hz}, \mathrm{CH}), 4.90(1 \mathrm{H}, \mathrm{d}, J=10.0 \mathrm{~Hz}, \mathrm{CH}), 4.67\left(1 \mathrm{H}, \mathrm{td}, J_{l}=\right.$ $\left.9.6 \mathrm{~Hz}, J_{2}=3.7 \mathrm{~Hz}, \mathrm{CH}\right), 4.01\left(1 \mathrm{H}, \mathrm{d}, J=18.0 \mathrm{~Hz}, \mathrm{CH}_{2}\right), 3.56\left(1 \mathrm{H}, \mathrm{d}, J=18.0 \mathrm{~Hz}, \mathrm{CH}_{2}\right)$, $3.40\left(2 \mathrm{H}, \mathrm{dd}, J_{l}=6.0 \mathrm{~Hz}, J_{2}=3.0 \mathrm{~Hz}, \mathrm{CH}_{2}\right), 3.35\left(3 \mathrm{H}, \mathrm{s}, \mathrm{CH}_{3}\right), 2.70\left(1 \mathrm{H}, \mathrm{dt}, J_{l}=14.6 \mathrm{~Hz}\right.$, $\left.J_{2}=10.0 \mathrm{~Hz}, \mathrm{CH}_{2}\right), 1.70-2.30\left(5 \mathrm{H}, \mathrm{m}, \mathrm{CH}_{2}\right) ;{ }^{13} \mathrm{C} \mathrm{NMR}\left(\mathrm{CDCl}_{3}, 300 \mathrm{MHz}\right): \delta 190.6,153.3$, 137.7, 134.5, 124.6, 124.0, 123.4, 123.2, 122.1, 110.8, 109.4, 58.1, 55.9, 55.0, 53.7, 32.5, 31.1, 26.3, 24.1; MS (CI) m/z $414\left(\mathrm{MH}^{+}\right)$; HRMS (CI) calcd for $(\mathrm{MH})^{+}, 414.1818$, found for $(\mathrm{MH})^{+}: 414.1815$.

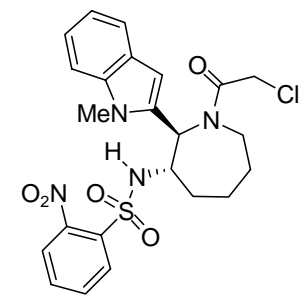

$\mathrm{N}-[1-(2-C h l o r o-a c e t y l)-2-(1-m e t h y l-1 \mathrm{H}-$ indol-2-yl)-azepan-3-yl]-2-nitro-

benzenesulfonamide 15

To a solution of diamine 7 (4.12 mmol, $1 \mathrm{~g})$ in THF (13 mL), $\mathrm{NaHCO}_{3}$ (3 eq., 12.3 mmol, $1.03 \mathrm{~g}$ ) was added at $0{ }^{\circ} \mathrm{C}$ under nitrogen. A solution of nosyl chloride (1.5 eq., 6.17 mmol, $1.37 \mathrm{~g})$ in THF $(13 \mathrm{~mL})$ was slowly added dropwise $(1 \mathrm{~mL} / \mathrm{h})$ at $0^{\circ} \mathrm{C}$. Stirring was maintained for $20 \mathrm{~h}$, keeping the reaction mixture at $0{ }^{\circ} \mathrm{C}$. The reaction mixture was cautiously diluted with $\mathrm{H}_{2} \mathrm{O}$ and extracted with $\mathrm{CH}_{2} \mathrm{Cl}_{2}(2 \times 50 \mathrm{~mL})$. The combined organic layers were dried $\left(\mathrm{MgSO}_{4}\right)$ and concentrated in vacuo. The residue was purified by chromatography (AcOEt/ $\mathrm{CH}_{3} \mathrm{CN}$ 95/5) to provide the protected azepane (yellow oil, $883 \mathrm{mg}$, $50 \%$ ). This product was dissolved in $\mathrm{CH}_{3} \mathrm{CN}\left(23 \mathrm{~mL}\right.$ ) at room temperature. $\mathrm{K}_{2} \mathrm{CO}_{3}$ (4 eq., 
$8.24 \mathrm{mmol}, 1.14 \mathrm{~g}$ ) was added and then chloroacetyl chloride (4 eq., $8.24 \mathrm{mmol}, 659 \mu \mathrm{L}$ ) was added dropwise. Stirring was maintained for $24 \mathrm{~h}$ at room temperature and the reaction mixture was concentrated in vacuo. The residue was dissolved in $\mathrm{CH}_{2} \mathrm{Cl}_{2}$ and $\mathrm{H}_{2} \mathrm{O}$ and extracted with $\mathrm{CH}_{2} \mathrm{Cl}_{2}(2 \times 50 \mathrm{~mL})$. The combined organic layers were dried $\left(\mathrm{MgSO}_{4}\right)$ and concentrated in vacuo to give an oily residue. The residue was purified by chromatography (AcOEt/cyclohexane 1/1) to provide chloroacetamide 15 (yellow oil, $729 \mathrm{mg}, 70 \%$ ). $[\alpha]_{\mathrm{D}}{ }^{20}$ 240 (c 1.1, $\mathrm{CH}_{2} \mathrm{Cl}_{2}$ ); IR (KBr) $v_{\max } 3322,2927,1637,1538,1442,1341,1163 \mathrm{~cm}^{-1} ;{ }^{1} \mathrm{H} \mathrm{NMR}$ $\left(\mathrm{CDCl}_{3}, 400 \mathrm{MHz}\right): \delta 7.63(1 \mathrm{H}, \mathrm{d}, J=7.7 \mathrm{~Hz}, \mathrm{CH}), 7.48(1 \mathrm{H}, \mathrm{d}, J=7.9 \mathrm{~Hz}, \mathrm{CH}), 7.42(1 \mathrm{H}$, $\mathrm{d}, J=7.8 \mathrm{~Hz}, \mathrm{CH}), 7.24(1 \mathrm{H}, \mathrm{d}, J=7.9 \mathrm{~Hz}, \mathrm{CH}), 7.18(1 \mathrm{H}, \mathrm{t}, J=7.5 \mathrm{~Hz}, \mathrm{CH}), 7.09(2 \mathrm{H}, \mathrm{m}$, 2CH), $6.98(1 \mathrm{H}, \mathrm{t}, J=7.7 \mathrm{~Hz}, \mathrm{CH}), 6.43(1 \mathrm{H}, \mathrm{s}, \mathrm{CH}), 6.06\left(1 \mathrm{H}, \mathrm{d}, J=8.4 \mathrm{~Hz}, \mathrm{SO}_{2} \mathrm{NH}\right), 5.73$ $(1 \mathrm{H}, \mathrm{d}, J=6.9 \mathrm{~Hz}, \mathrm{CH}), 4.33\left(1 \mathrm{H}, \mathrm{dd}, J_{1}=8.4 \mathrm{~Hz}, J_{2}=6.9 \mathrm{~Hz}, \mathrm{CH}\right), 4.10\left(2 \mathrm{H}, \mathrm{s}, \mathrm{CH}_{2}\right), 3.50$ $\left(1 \mathrm{H}, \mathrm{bd}, J=13.5 \mathrm{~Hz}, \mathrm{CH}_{2}\right), 3.39\left(3 \mathrm{H}, \mathrm{s}, \mathrm{CH}_{3}\right), 3.14\left(1 \mathrm{H}, \mathrm{td}, J_{1}=13.5 \mathrm{~Hz}, J_{2}=2.2 \mathrm{~Hz}, \mathrm{CH}_{2}\right)$, $2.24\left(1 \mathrm{H}, \mathrm{dd}, J_{l}=13.6 \mathrm{~Hz}, J_{2}=7.2 \mathrm{~Hz}, \mathrm{CH}_{2}\right), 2.03\left(1 \mathrm{H}, \mathrm{m}, \mathrm{CH}_{2}\right), 1.50-1.90\left(4 \mathrm{H}, \mathrm{m}, \mathrm{CH}_{2}\right)$; ${ }^{13} \mathrm{C}$ NMR $\left(\mathrm{CDCl}_{3}, 100 \mathrm{MHz}\right): \delta 167.8,148.9,137.5,136.5,134.2,132.7,132.0,130.2,126.7$, $124.5,122.7,120.0,119.1,109.7,102.2,60.0,55.0,43.8,40.9,35.1,29.8,25.3 ; \mathrm{MS}(\mathrm{CI}) \mathrm{m} / \mathrm{z}$ $505.1\left(\mathrm{MH}^{+}\right)$; HRMS $(\mathrm{CI})$ calcd for $(\mathrm{MH})^{+}, 505.1312$, found for $(\mathrm{MH})^{+}: 505.1315$.

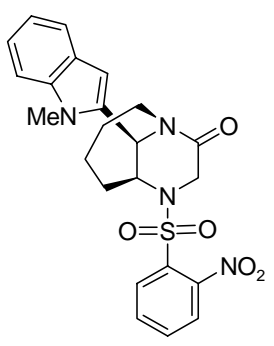

10-(1-Methyl-1H-indol-2-yl)-7-(2-nitrobenzenesulfonyl)-1,7-diaza bicyclo[4.3.1]decan-9-one $\mathbf{1 6}$

To a solution of chloroacetamide $15(0.129 \mathrm{mmol}, 65 \mathrm{mg})$ in $\mathrm{CH}_{3} \mathrm{CN}(2 \mathrm{~mL}), \mathrm{K}_{2} \mathrm{CO}_{3}$ (2 eq., $0.258 \mathrm{mmol}, 36 \mathrm{mg}$ ) and $\mathrm{KI}(2$ eq., $0.258 \mathrm{mmol}, 43 \mathrm{mg}$ ) were added at room temperature under nitrogen. The reaction mixture was stirred for $24 \mathrm{~h}$ at reflux and concentrated in vacuo. The residue was diluted in $\mathrm{CH}_{2} \mathrm{Cl}_{2}$ and $\mathrm{H}_{2} \mathrm{O}$ and extracted with $\mathrm{CH}_{2} \mathrm{Cl}_{2}$ $(2 \times 10 \mathrm{~mL})$. The combined organic layers were dried $\left(\mathrm{MgSO}_{4}\right)$ and concentrated in vacuo. The residue was purified by chromatography (AcOEt/cyclohexane 7/3) to provide piperazinone 16 (yellow oil, $30 \mathrm{mg}, 50 \%)$. $[\alpha]_{\mathrm{D}}{ }^{20}+285\left(\mathrm{c} 0.6, \mathrm{CH}_{2} \mathrm{Cl}_{2}\right)$; IR $(\mathrm{KBr}) v_{\max } 2944$, $1679,1543,1470,1418,1372,1166,1127,1067 \mathrm{~cm}^{-1} ;{ }^{1} \mathrm{H} \mathrm{NMR}\left(\mathrm{CDCl}_{3}, 400 \mathrm{MHz}\right): \delta 8.06$ $\left(1 \mathrm{H}, \mathrm{dd}, J_{l}=4.2 \mathrm{~Hz}, J_{2}=2.6 \mathrm{~Hz}, \mathrm{CH}\right), 7.71\left(2 \mathrm{H}, \mathrm{td}, J_{l}=4.2 \mathrm{~Hz}, J_{2}=1.1 \mathrm{~Hz}, 2 \mathrm{CH}\right), 7.61(2$ $\mathrm{H}, \mathrm{m}, 2 \mathrm{CH}), 7.30(2 \mathrm{H}, \mathrm{m}, 2 \mathrm{CH}), 7.16\left(1 \mathrm{H}, \mathrm{td}, J_{1}=7.8 \mathrm{~Hz}, J_{2}=1.1 \mathrm{~Hz}, \mathrm{CH}\right), 6.70(1 \mathrm{H}, \mathrm{s}$, $\mathrm{CH}), 5.00(1 \mathrm{H}, \mathrm{s}, \mathrm{CH}), 4.92(1 \mathrm{H}, \mathrm{s}, \mathrm{CH}), 4.32(2 \mathrm{H}, \mathrm{m}, \mathrm{CH}), 3.97\left(1 \mathrm{H}, \mathrm{d}, J=14.2 \mathrm{~Hz}, \mathrm{CH}_{2}\right)$, $3.63\left(3 \mathrm{H}, \mathrm{s}, \mathrm{CH}_{3}\right), 2.52\left(1 \mathrm{H}, \mathrm{dd}, J_{l}=13.7 \mathrm{~Hz}, J_{2}=8.4 \mathrm{~Hz}, \mathrm{CH}_{2}\right), 2.35\left(2 \mathrm{H}, \mathrm{m}, \mathrm{CH}_{2}\right), 1.72(2$ $\left.\mathrm{H}, \mathrm{m}, \mathrm{CH}_{2}\right), 1.36\left(2 \mathrm{H}, \mathrm{m}, \mathrm{CH}_{2}\right) ;{ }^{13} \mathrm{C} \mathrm{NMR}\left(\mathrm{CDCl}_{3}, 100 \mathrm{MHz}\right): \delta 169.9,148.8,138.0,134.8$, 132.3, 132.0, 131.6, 131.1, 127.2, 124.8, 123.3, 121.5, 120.6, 109.6, 103.3, 56.5, 56.2, 48.5, 41.2, 35.6, 30.4, 27.3, 20.4; MS (CI) m/z $469\left(\mathrm{MH}^{+}\right)$; HRMS (CI) calcd for $(\mathrm{MH})^{+}, 469.1546$, found for $(\mathrm{MH})^{+}: 469.1547$. 


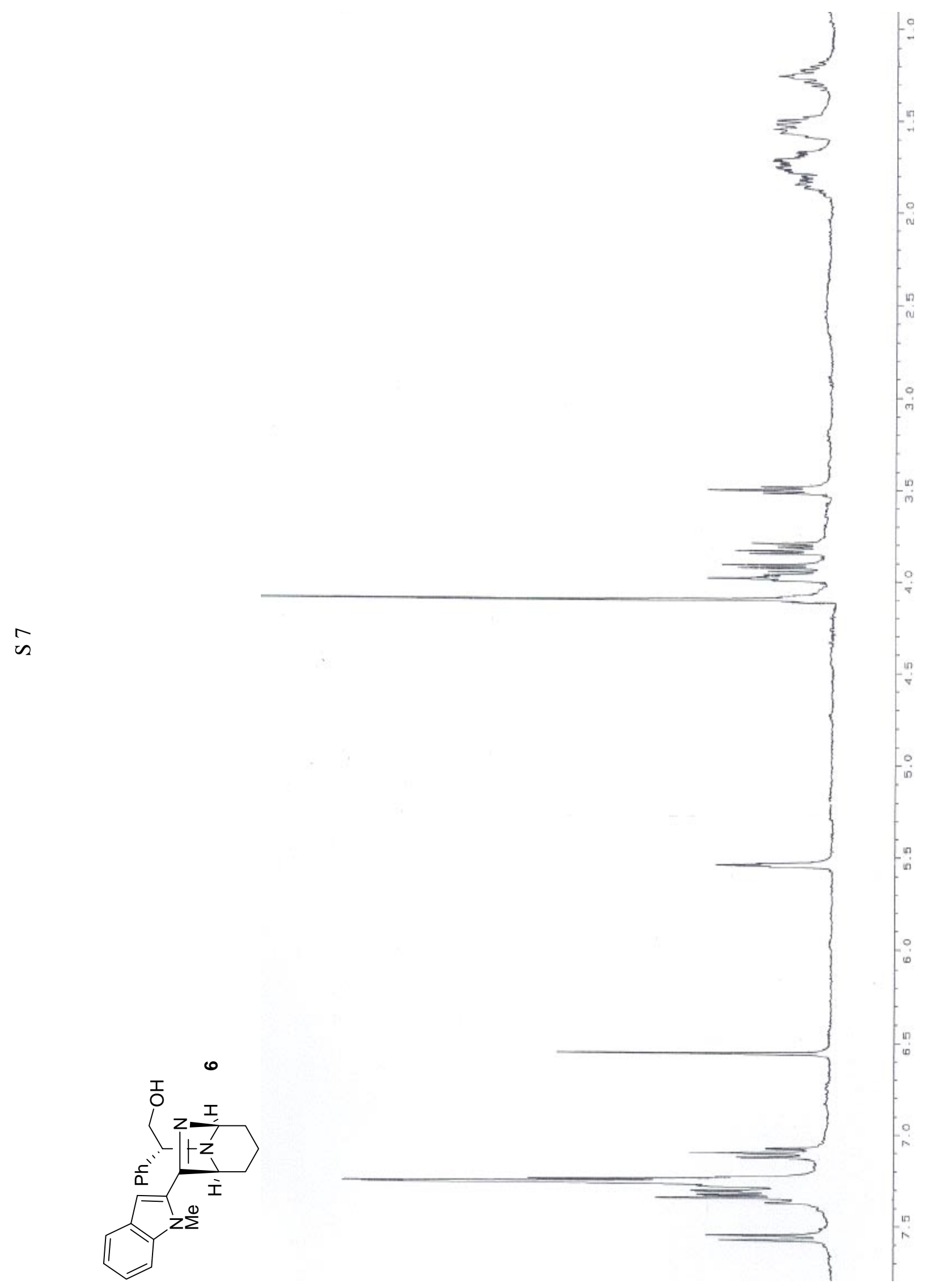




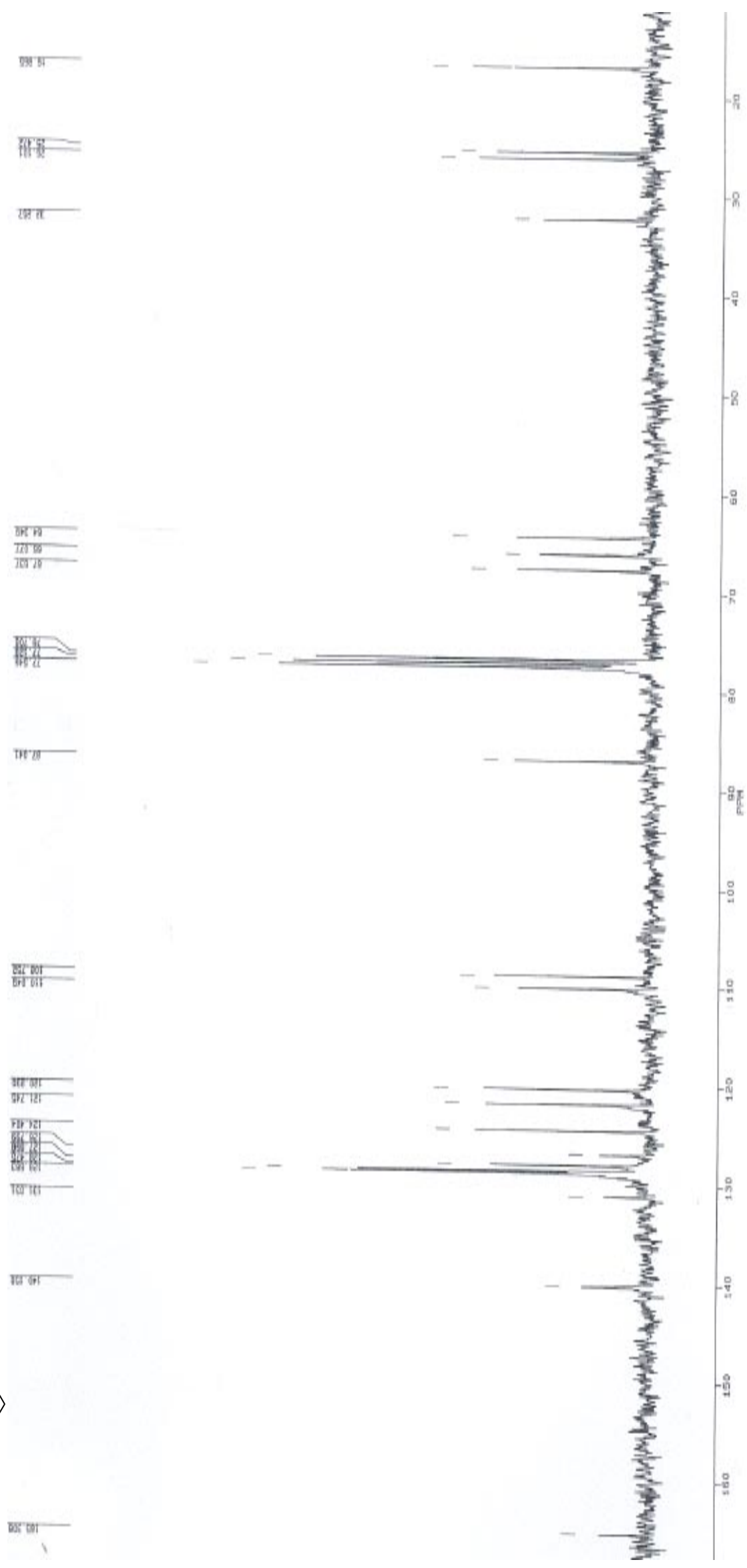




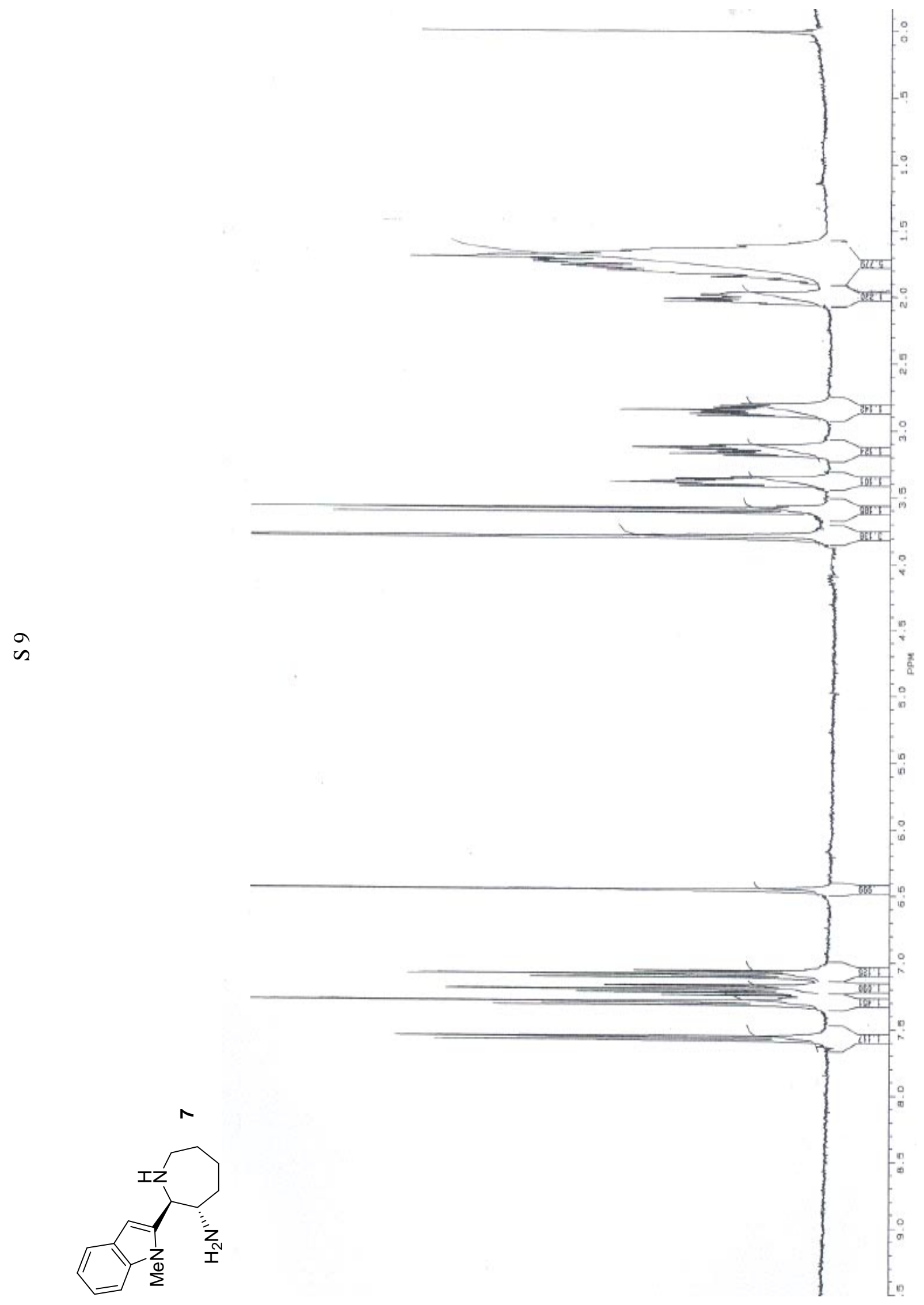



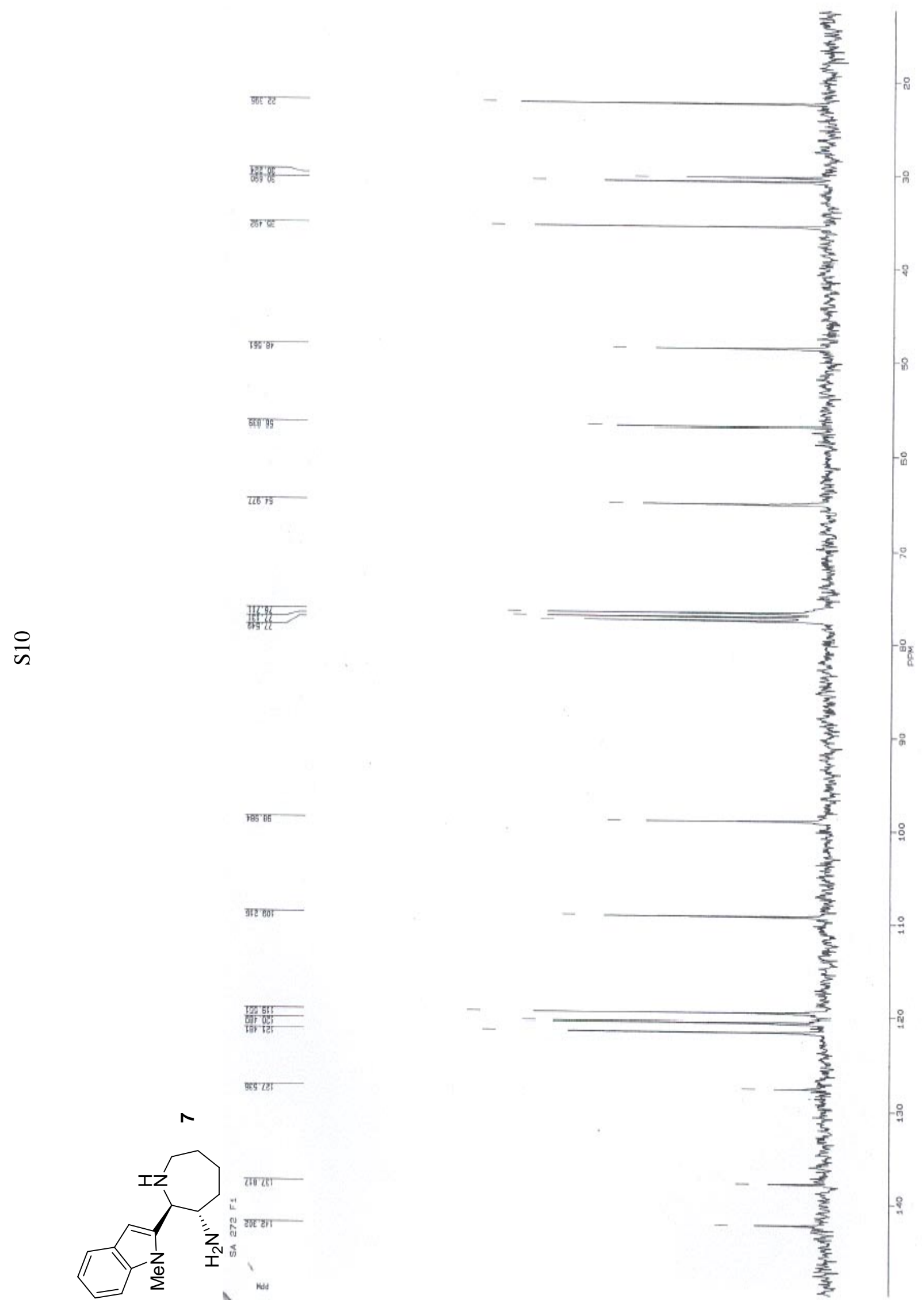


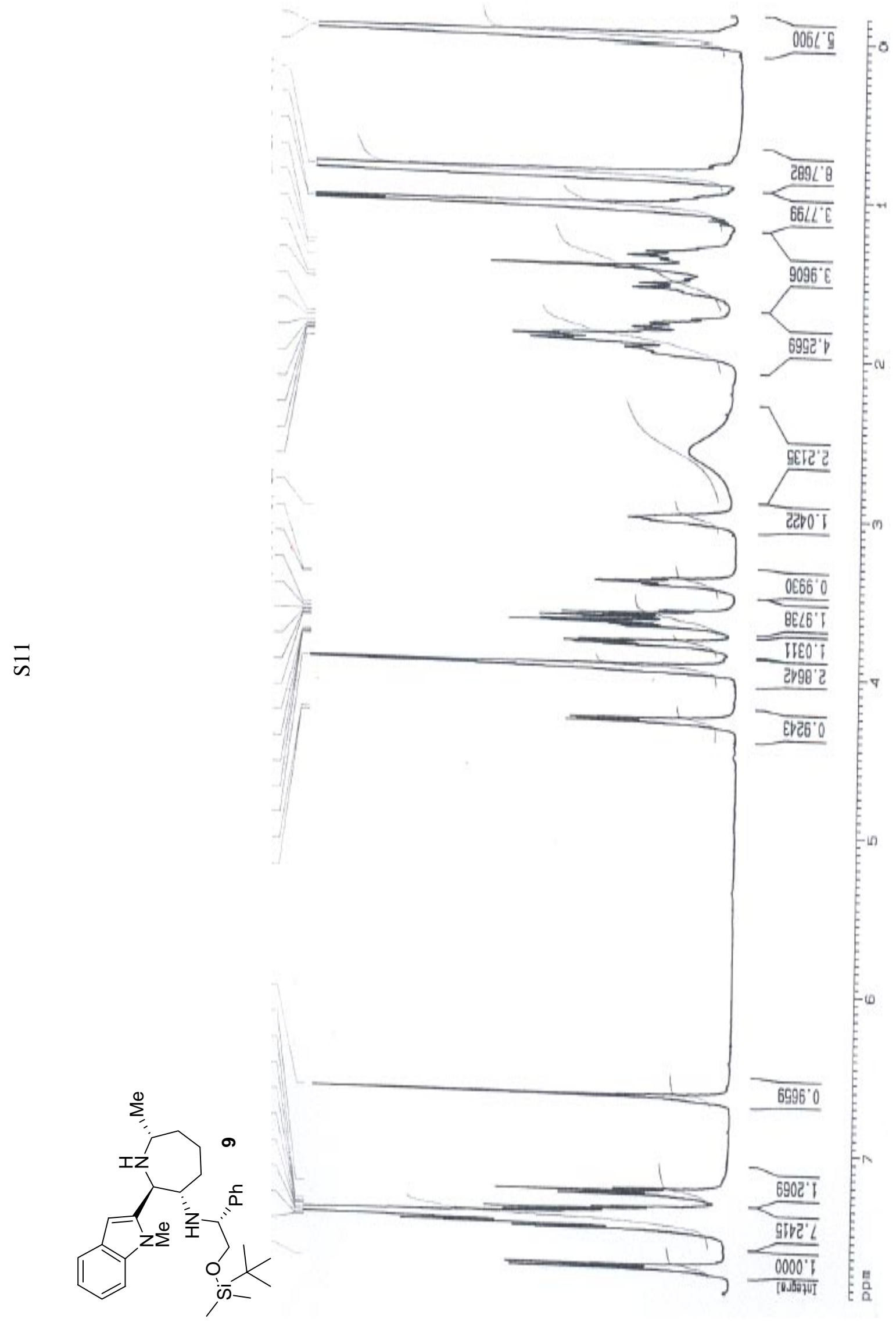




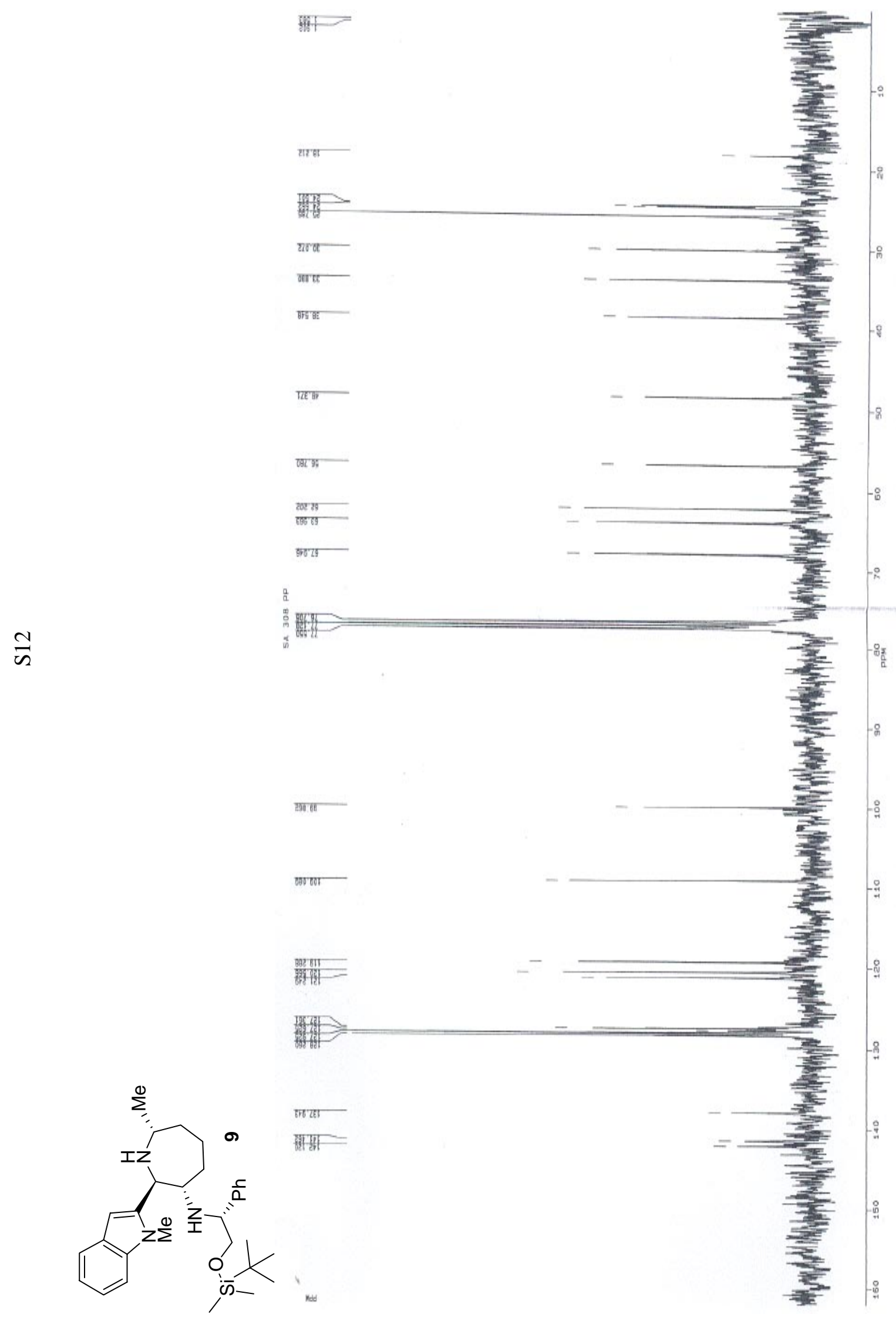




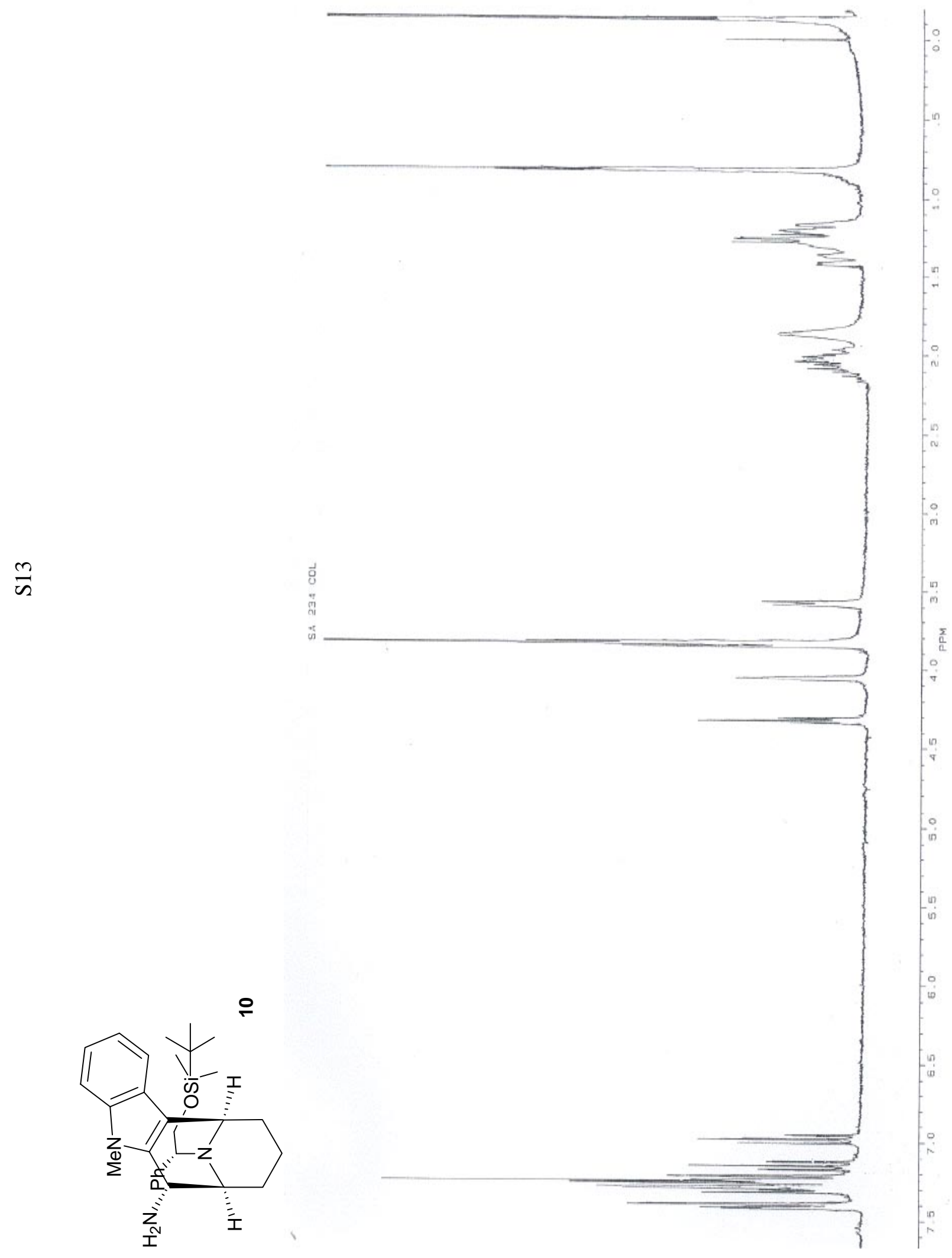




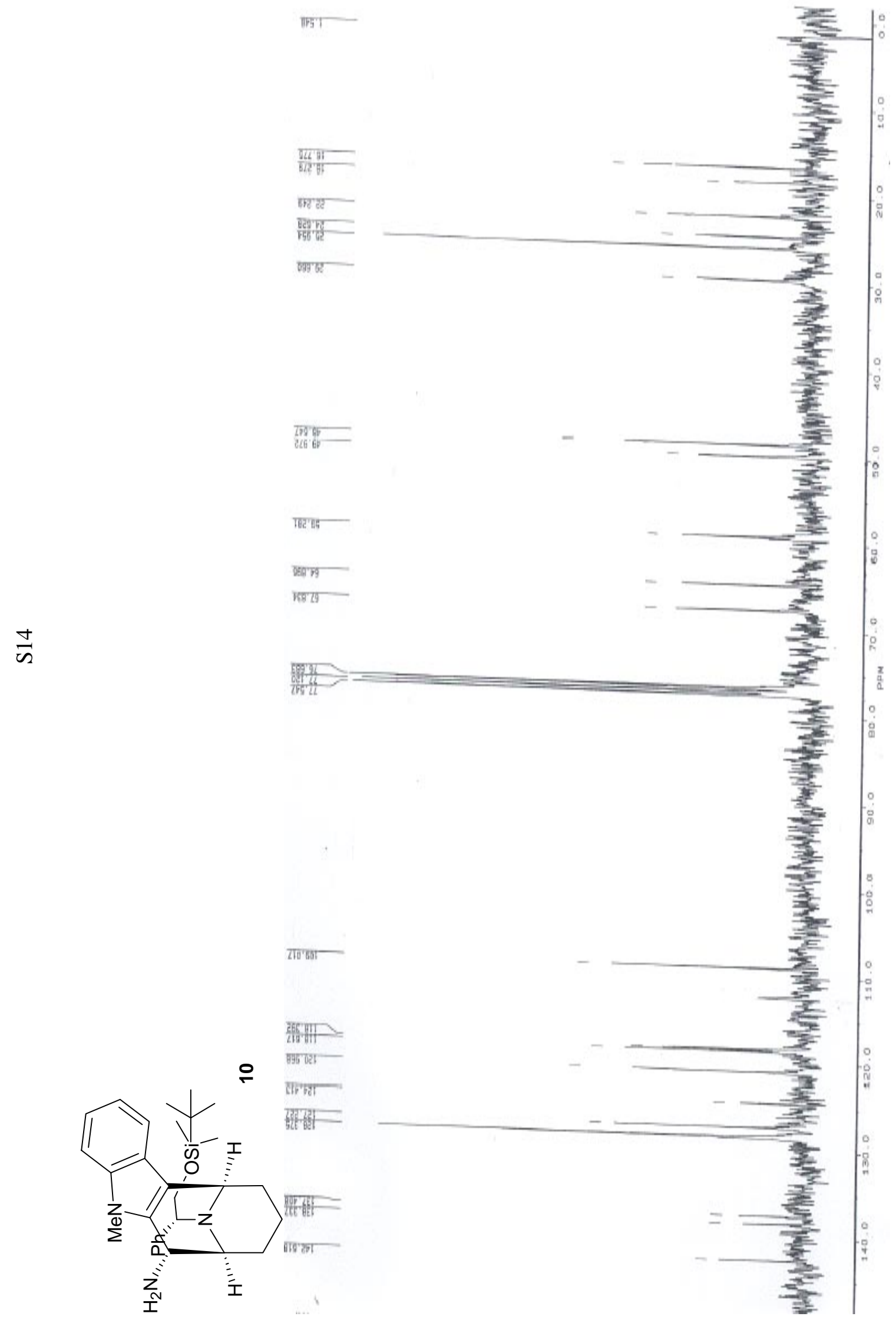


$\frac{n}{n}$

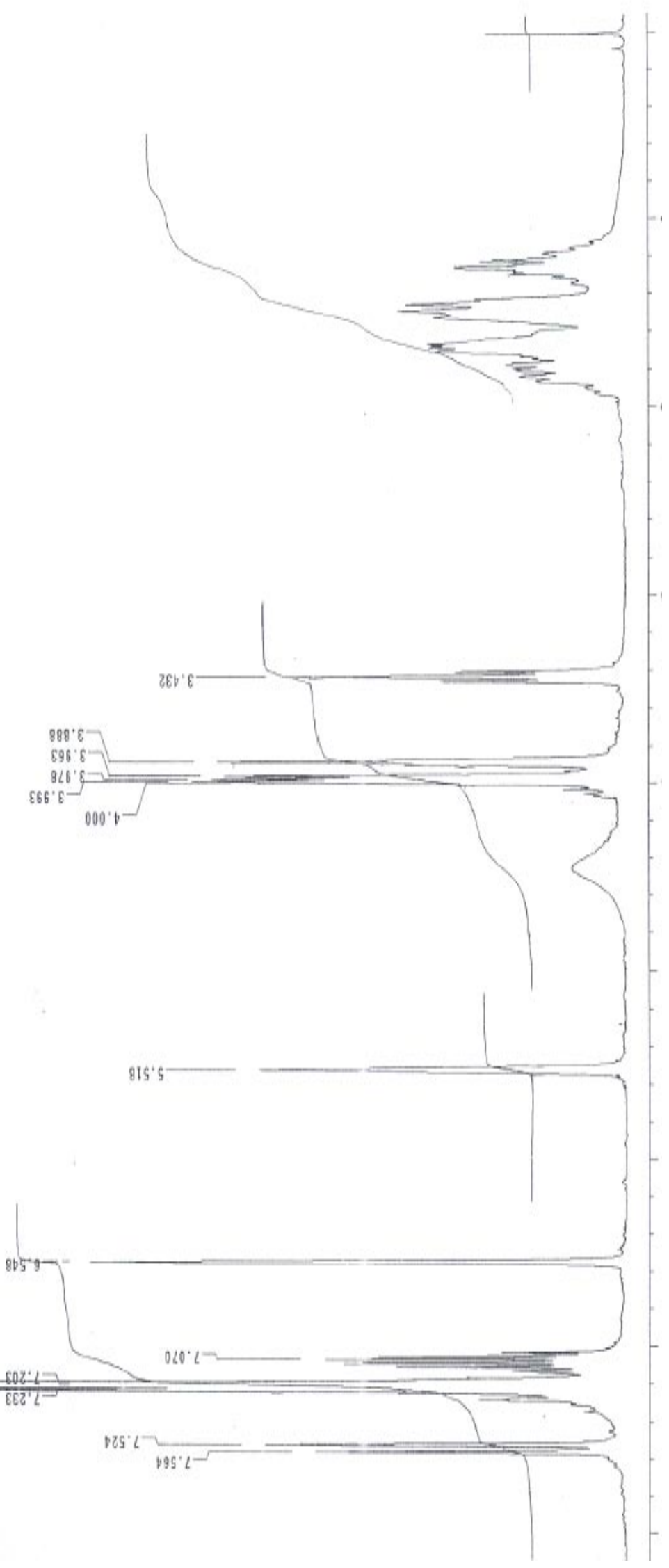




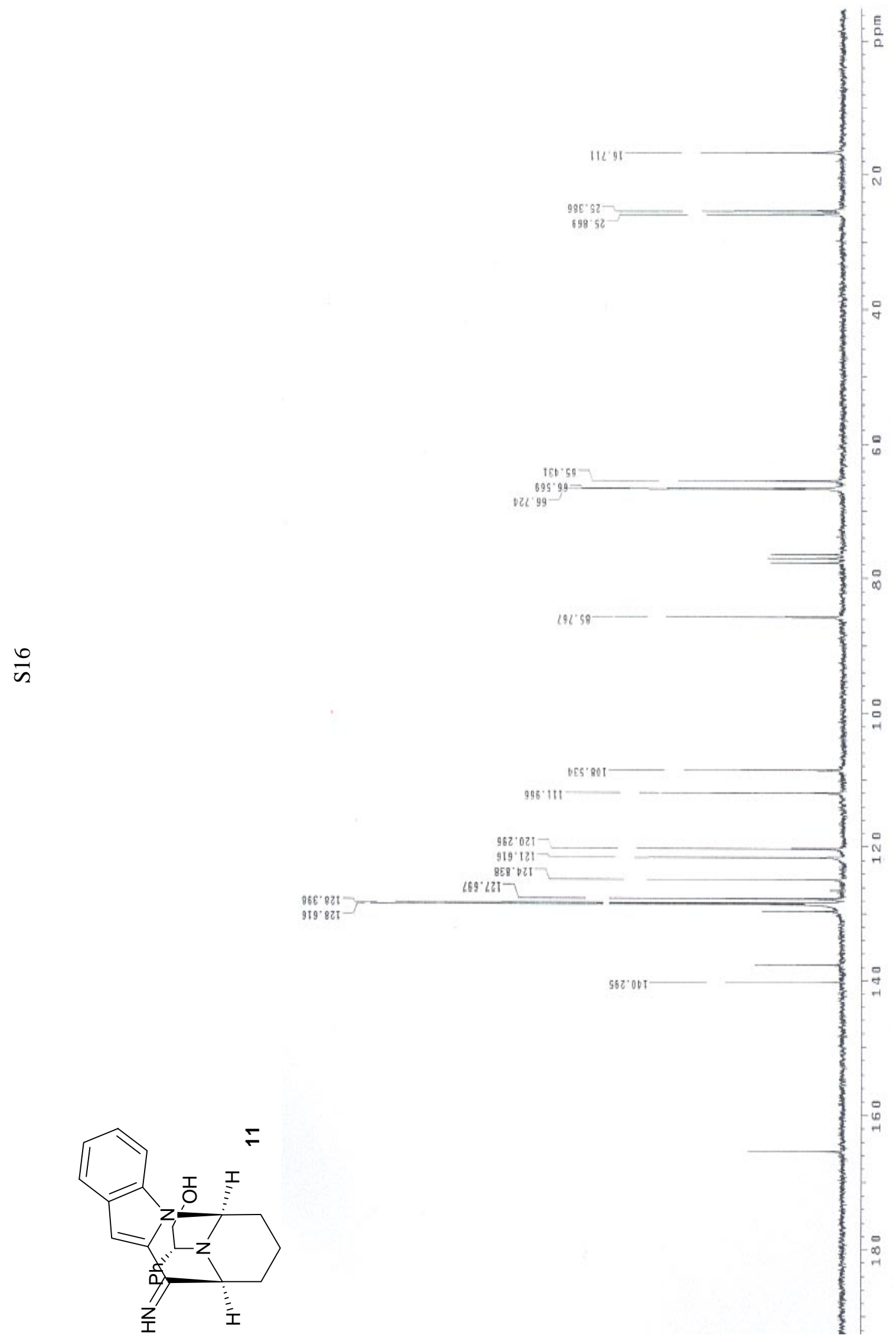




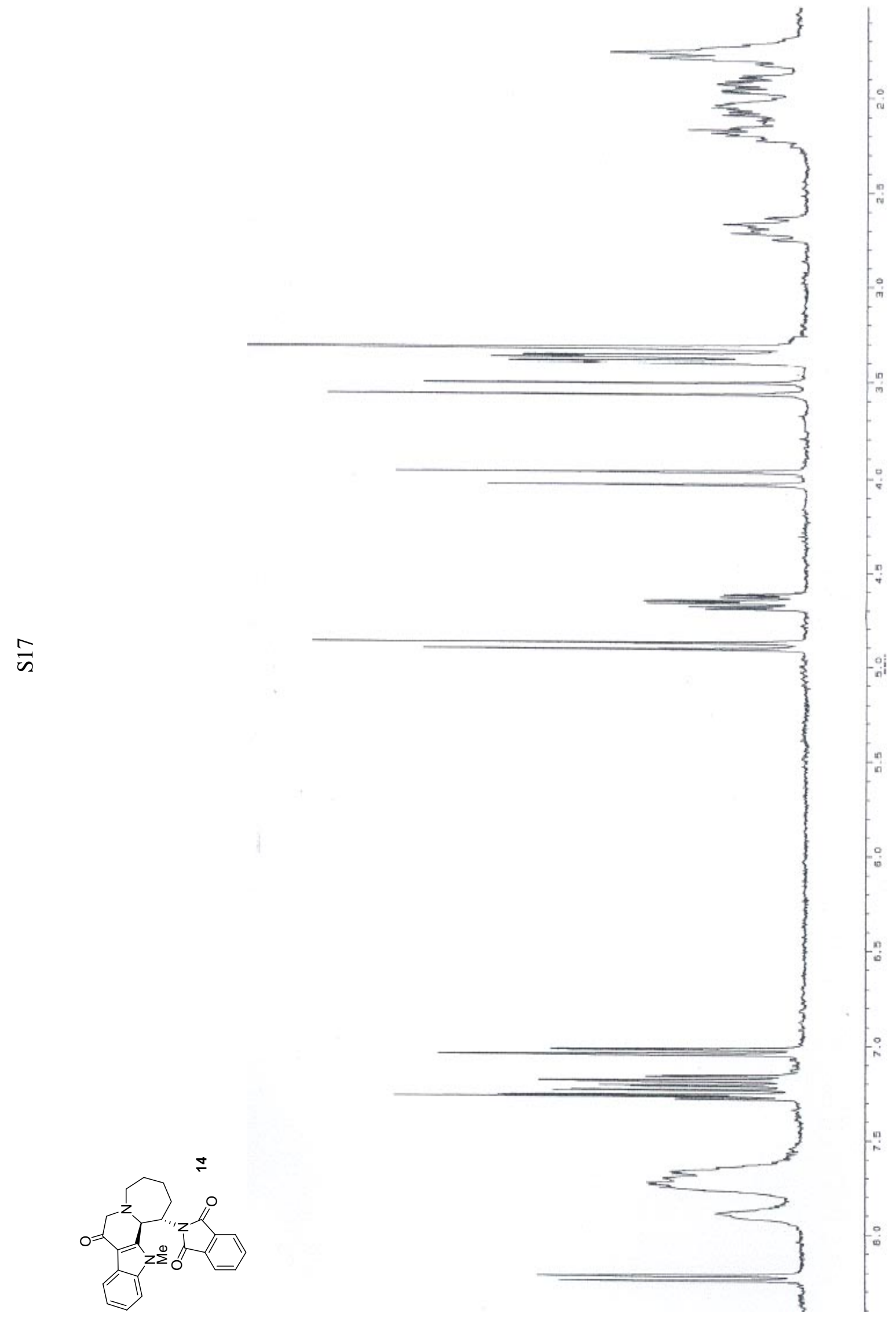




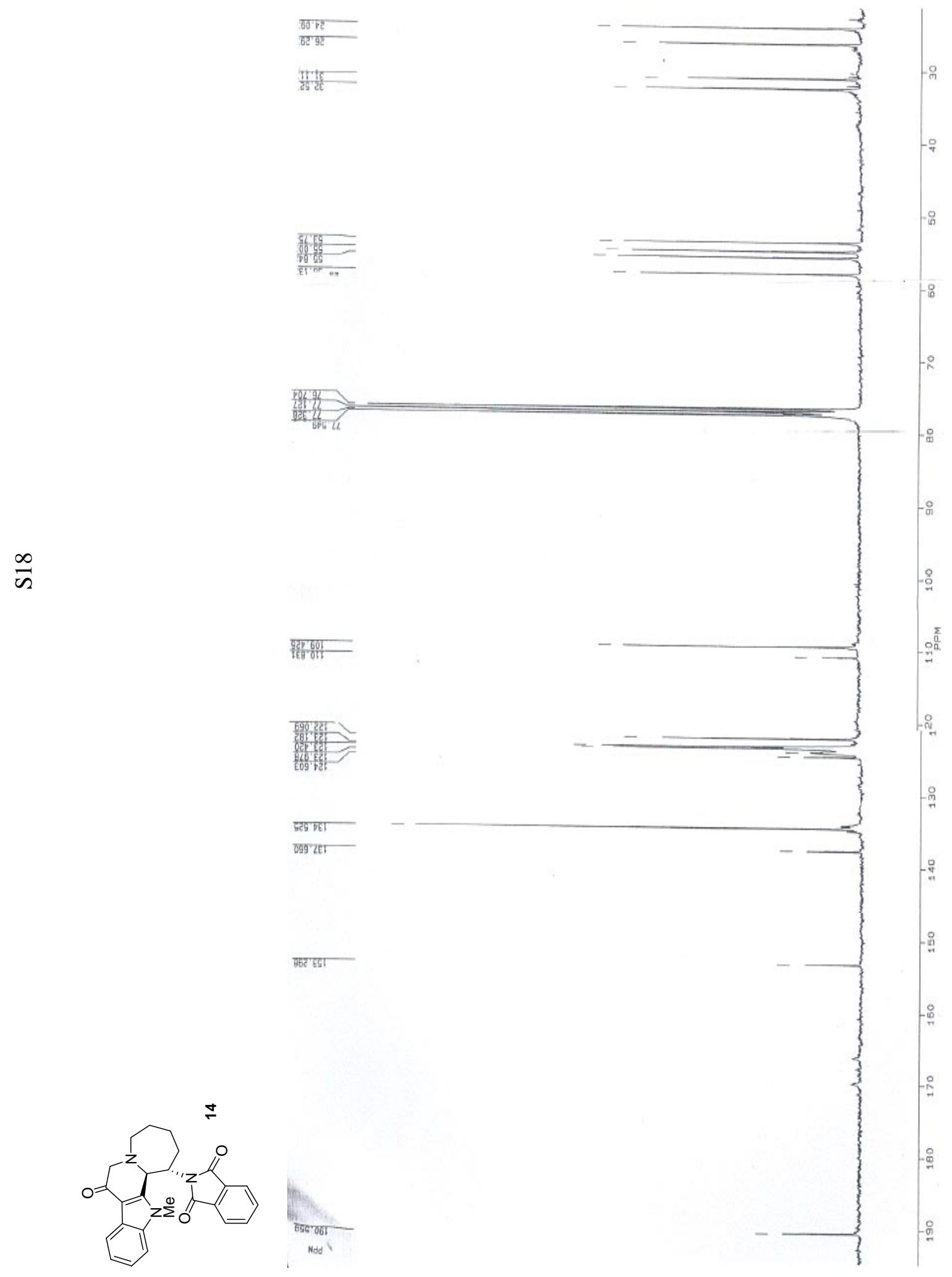




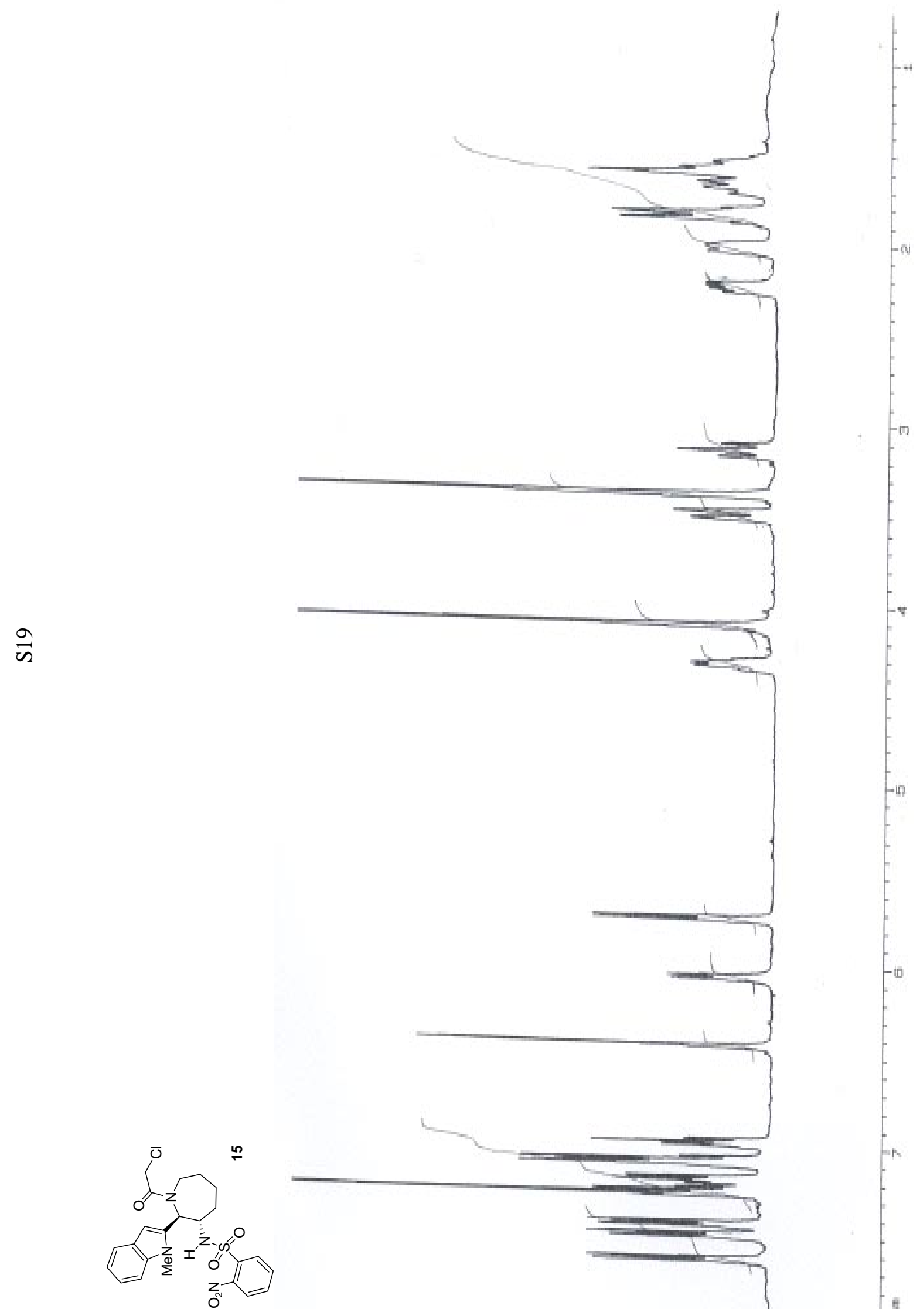



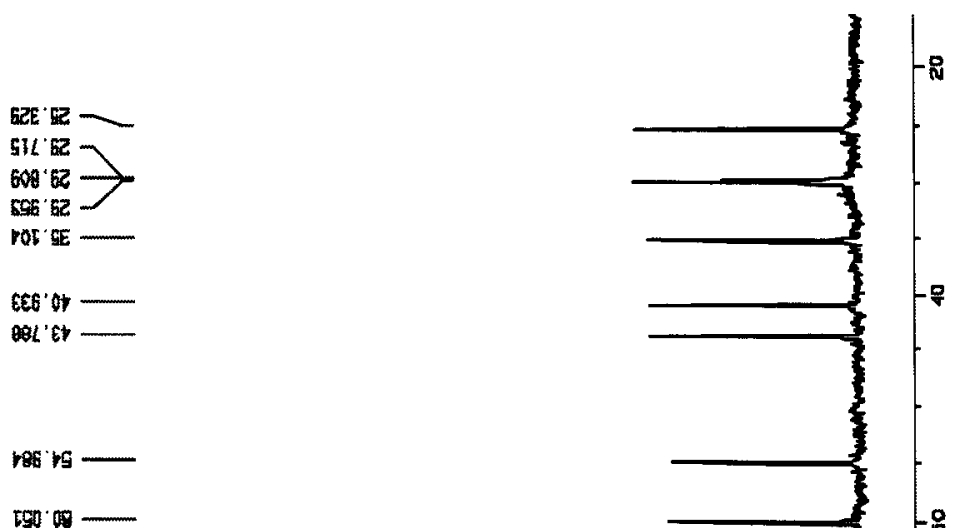

t5n of -

$8002 \mathrm{SL}$

द्वा' $L$

Et? $U$

ชิ
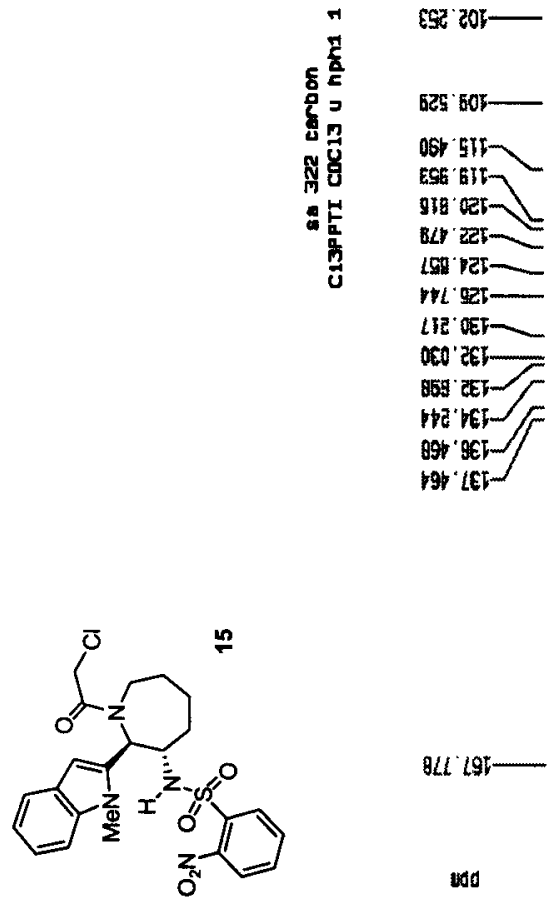

gul 2 So

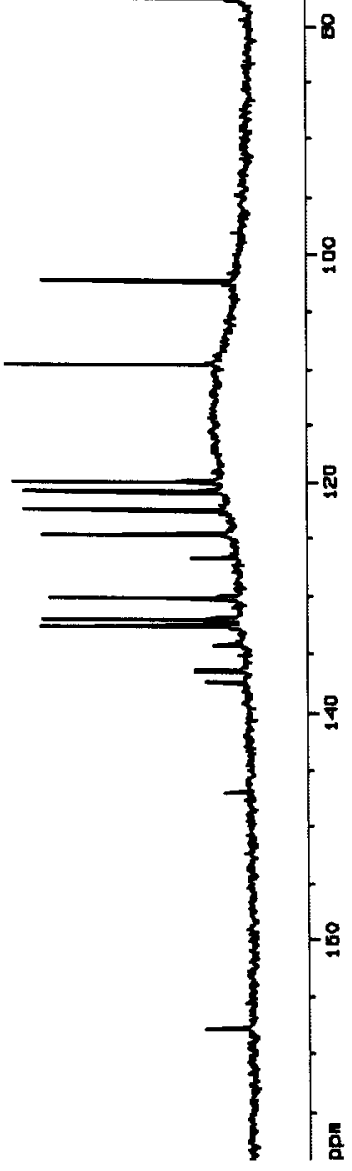




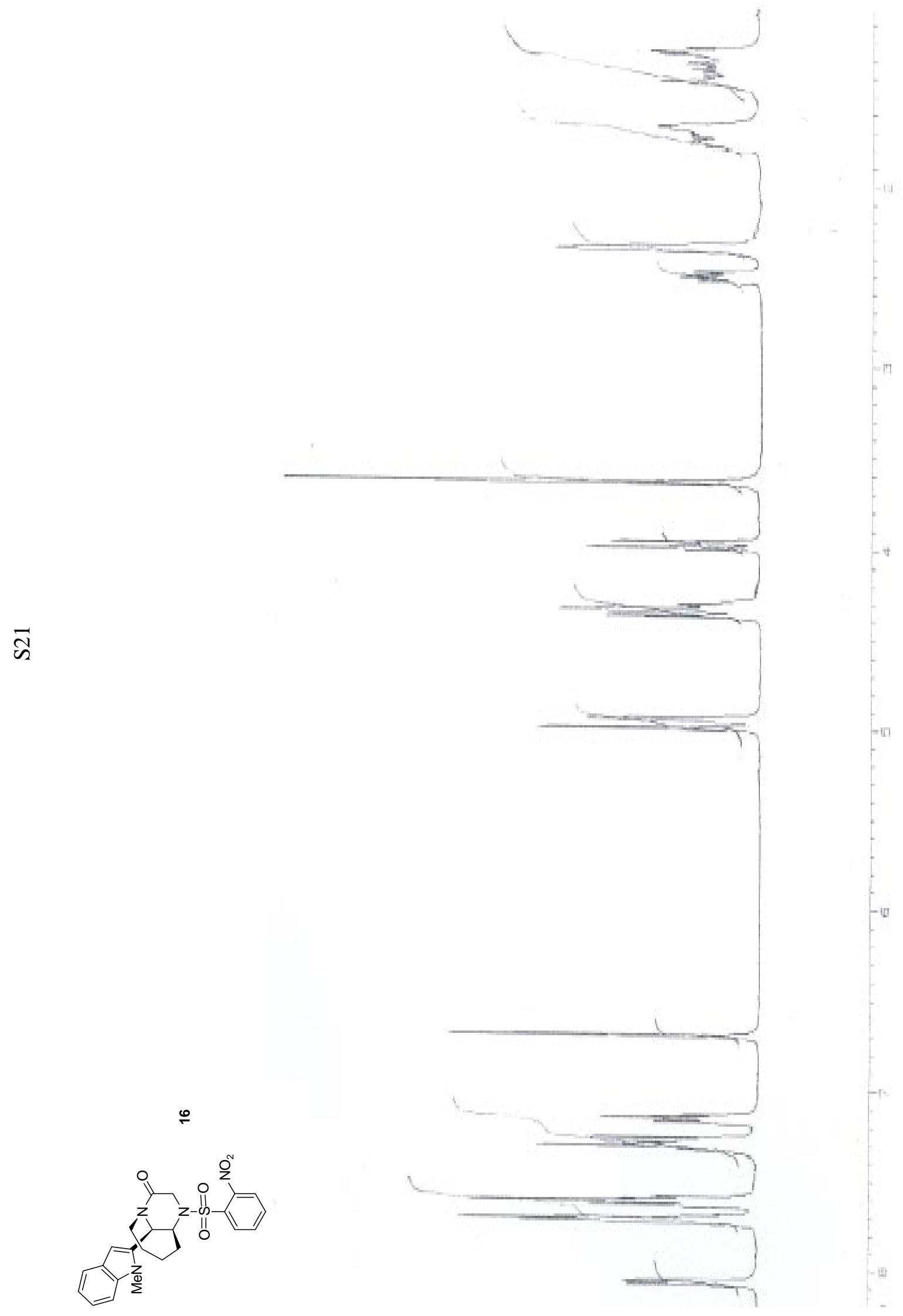




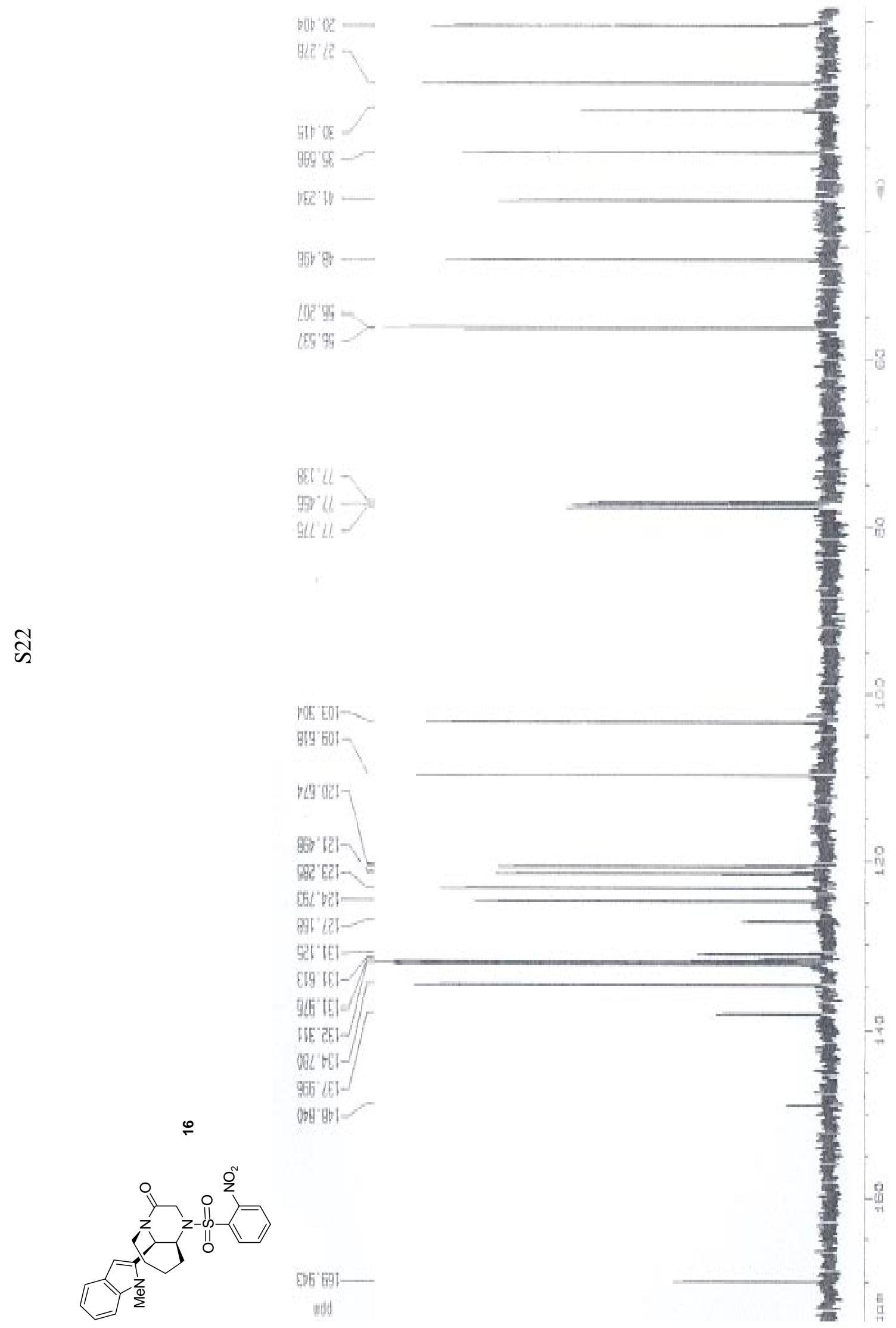

